\title{
Mixed Numerologies Interference Analysis and Inter-Numerology Interference Cancellation for Windowed OFDM Systems
}

\author{
Xiaoying Zhang, Lei Zhang, Pei Xiao, Senior Member, IEEE, Dongtang Ma, Jibo Wei, Yu Xin
}

\begin{abstract}
Extremely diverse service requirements are one of the critical challenges for the upcoming fifth-generation (5G) radio access technologies. As a solution, mixed numerologies transmission is proposed as a new radio air interface by assigning different numerologies to different subbands. However, coexistence of multiple numerologies induces the inter-numerology interference (INI), which deteriorates the system performance. In this paper, a theoretical model for INI is established for windowed orthogonal frequency division multiplexing (W-OFDM) systems. The analytical expression of the INI power is derived as a function of the channel frequency response of interfering subcarrier, the spectral distance separating the aggressor and the victim subcarrier, and the overlapping windows generated by the interferer's transmitter windows and the victim's receiver window. Based on the derived INI power expression, a novel INI cancellation scheme is proposed by dividing the INI into a dominant deterministic part and an equivalent noise part. A soft-output ordered successive interference cancellation (OSIC) algorithm is proposed to cancel the dominant interference, and the residual interference power is utilized as effective noise variance for the calculation of loglikelihood ratios (LLRs) for bits. Numerical analysis shows that the INI theoretical model matches the simulated results, and the proposed interference cancellation algorithm effectively mitigates the INI and outperforms the state-of-the-art W-OFDM receiver algorithms.
\end{abstract}

Index Terms-Windowed-OFDM (W-OFDM), internumerology interference, interference cancellation, multi-service, mixed numerologies

\section{INTRODUCTION}

The upcoming fifth-generation (5G) wireless communication systems need to support a vast variety of user cases with diverse requirements. Three major services with extremely diverse communication requirements are expected to be deployed in 5G multi-service network, i.e., enhancedmobile broadband (eMBB), ultra reliable and low latency communications (URLLC) and massive machine type communications (mMTC) [1]. The mMTC application prefers smaller subcarrier spacing and thus larger symbol duration to support

Copyright (c) 2015 IEEE. Personal use of this material is permitted. However, permission to use this material for any other purposes must be obtained from the IEEE by sending a request to pubs-permissions@ieee.org

This work was supported in part by the Research Found of National University of Defense Technology under Grant ZK17-03-13.

X. Zhang, D. Ma and J. Wei are with the Institute of Electronic Science, National University of Defense Technology, China (e-mail: zhangxiaoying@nudt.edu.cn; Dongtangma@nudt.edu.cn; wjbhw@nudt.edu.cn).

L. Zhang is with the Shchool of Engineering, University of Glasgow, U.K. (e-mail: Lei.Zhang@glasgow.ac.uk).

P. Xiao is with the center of communication systems research (CCSR), University of Surrey, U.K. (e-mail: P.xiao@surrey.ac.uk).

Yu Xin is with the ZTE Corporation, China (e-mail: xin.yu@zte.com.cn). delay-tolerant devices with up to $10^{6}$ devices $/ \mathrm{km}^{2}$ connection density [2]. On the other hand, URLLC needs high reliability and low end-to-end delay down to less than 1 millisecond, which requires a larger subcarrier spacing to meet stringent latency requirements [3]. eMBB is expected to support 20 Gbps downlink peak data rate and 10 Gbps uplink peak data rate for some 5G applications [4], whose performance is significantly affected by channel characteristics. For instance, in the presence of the fast fading channel, large subcarrier spacing is generally preferable for highly mobile users to counteract the Doppler spread, while smaller subcarrier spacing can achieve higher robustness for the channel with long delay spread.

To embrace such diverse service requirements, the traditional uniform time/frequency resources blocks definition, single numerology and subframe format adopted by the 4G Long Term Evolution (LTE) might not be suitable any more. Since the multitude of heterogeneous services should be provided simultaneously by a common underlying physical layer, separate radio design for each service is not practical due to the unfeasible cost and complexity. In addition, it is cumbersome to design a one-fits-all solution to meet all service requirements [5]. Therefore, mixed numerologies are proposed for the new radio air interface to optimize the individual service, which can be supported by the time domain multiplexing or frequency domain multiplexing. For the conventional orthogonal frequency division multiplexing (OFDM) which is well-localized in time domain, aligning different numerologies in time domain can maintain the orthogonality between the consecutive blocks [6]. However, multiplexing services in frequency domain have better forward compatibility and inclusive support for different latency services compared with the time domain multiplexing counterpart [5]. Thus, a viable and mostly accepted way to cater for diverse services is to divide the bandwidth into several subbands and assign different numerologies to different subbands [7]. Assigning different numerologies in the frequency domain has been absorbed as the commonly accepted simulation evaluation cases in the Third Generation Partnership Project (3GPP) discussions [8]. Although the subcarriers with the same numerology are orthogonal to each other, subcarriers with different numerologies, e.g., different subcarrier spacing and length of cyclic prefix (CP), may interfere with each other, especially for those adjacent ones. Hence, a severe problem of frequency domain multiplexing mixed numerologies transmission is the performance degradation caused by internumerology interference (INI). In order to mitigate INI, insert- 
ing guard bands (GB) between subbands may be a solution. However, it comes at a cost of spectrum efficiency reduction. In [7], the authors reported a field trial using adaptive coding and modulation for mixed numerologies transmission in the filtered-OFDM (f-OFDM) systems. However, only the lineof-sight transmission scenario is considered and no theoretical analysis for INI is given. A precoding scheme is proposed in [5] to suppress the inter-service interference for universal filtered multi-carrier (UFMC) systems. However, to pre-cancel the interference, the transmitter requires accurate channel state information and noise variance feedback from the receiver, which may not be practical assumption in many situations. A multi-service interference model is proposed for f-OFDM and UFMC systems in [9] and [10]. Since the interference model is derived based on the subband filtered multi-carrier systems, it can not be directly extended to the windowed-OFDM (WOFDM) systems [11].

Physical layer waveform is the basic element to support the multi-service of $5 \mathrm{G}$ and has critical impact on the INI. All the promising waveforms, e.g., W-OFDM [12], f-OFDM [13], UFMC [14], filter bank multi-carrier (FBMC) [15], and generalized frequency-division multiplexing (GFDM) [16], have different advantages and drawbacks, none of them can address all the requirements of 5G [17]. According to the recent agreement reached by 3GPP [18], [19], OFDM is still the base for the new waveform for $5 \mathrm{G}$, especially in the broadband scenario, and the backward-compatibility and transparency of transceiver are highlighted for the waveform candidates. W-OFDM preserves the core receiver structure of OFDM and allows transparent transmitter and receiver windowing procedure. Compared with filtering-type technique, $\mathrm{W}$ OFDM is associated with relatively less complex windowing for spectral confinement and has little or no peak-to-average power ratio (PAPR) overhead [20]. Therefore, it has drawn significant attention from academia and industry. The authors in [21] investigated the performance of W-OFDM in the presence of carrier frequency offset and timing offset, the study revealed that W-OFDM offers robustness to asynchronism. On the other hand, great efforts have been taken to improve the performance and flexibility for W-OFDM. Window functions were discussed and optimized in [22]. The receiver windowing was considered along with the transmitter windowing in [23] to reject the adjacent channel interference and limit the outof-band emission (OOBE), respectively. To alleviate the intersymbol interference (ISI) induced by the reduced CP length while maintain the OOBE suppression, a time-asymmetric windowing scheme is proposed in [11]. The authors of [24] proposed the windowing scheme for optimal time-frequency concentration for W-OFDM systems. A flexible windowing method was proposed in [25] and further extended in [17] to balance the OOBE and robustness against channel delay spread.

To sum up, W-OFDM is a competitive waveform candidate which has been intensively studied. However, the INI for WOFDM has not been analytically discussed in the context of mixed numerologies transmission. By using numerical simulation, [26] and [27] evaluated the average signal-tointerference ratio (SIR) of the mixed numerologies interference based on W-OFDM systems. To the best knowledge of the authors, no general analytical expression of the INI has been given for W-OFDM systems in the literature to guide the $5 \mathrm{G}$ system design and parameters selection. In addition, it is still not yet clear how the transmitter/receiver windows affect the INI quantitatively, although it is intuitively understood that windows with larger roll-off length lead to better capability of interference resistance. Besides the waveform and window design and analysis, effective interference cancellation algorithm is critical to achieve an acceptable performance, especially for those subcarriers suffering serious INI. However, such an algorithm has not been proposed for W-OFDM systems. Our research is motivated to solve these problems.

The main contributions of this paper are listed as follows.

- A theoretical INI model is first built for W-OFDM systems in the presence of mixed numerologies transmission in this paper. Based on the INI model, analytical expressions are derived to directly evaluate the INI power for W-OFDM systems in the frequency selective fading channels. According to the derived expressions, the power of INI caused by a given interfering subcarrier on a victim subcarrier depends on their spectral distance, the channel frequency response of interferer and the overlapping windows generated by the aggressor's transmitter windows and victim's receiver window. The expressions explain explicitly how the transmitter and receiver windows affect the INI level, and they can be applied to OFDM as a special case of W-OFDM.

- With the established analytical model, the properties of INI for W-OFDM systems are discussed and analyzed, which give useful insights for the system design and window optimization. It is unveiled that compared with OFDM, the significant SIR gain of W-OFDM can only be obtained for the subcarriers with relatively large spectral distance, while for edge subcarriers with non-sufficient GB, severe INI are imposed for W-OFDM systems. This phenomenon motivates to the next contribution.

- An INI power aided interference cancellation algorithm is proposed in this paper. Based on the derived analytical expressions for INI power, the INI is divided into a dominant deterministic part and an equivalent noise part. A soft-output ordered successive interference cancellation (OSIC) detector is proposed for the joint detection of the dominant victim subcarriers and interfering subcarriers to cancel the major INI, and the non-dominant interference power is utilized as effective noise variance for the calculation of LLRs of coded bits, which are fed to the soft decoder to improve the system performance.

The rest of the paper is organized as follows. In Section II, the W-OFDM system model under investigation is presented. Section III derives the system model for mixed numerologies transmission. In Section IV, analytical expressions for the INI power are presented. The INI cancellation algorithm is proposed in Section V. Section VI shows the simulation and analysis results. Finally, conclusions are drawn in Section VII.

Notations: Superscripts $(\cdot)^{T}$ and $(\cdot)^{H}$ denote transpose and conjugate transpose, respectively. $\operatorname{diag}(\mathbf{x})$ is a diagonal matrix 


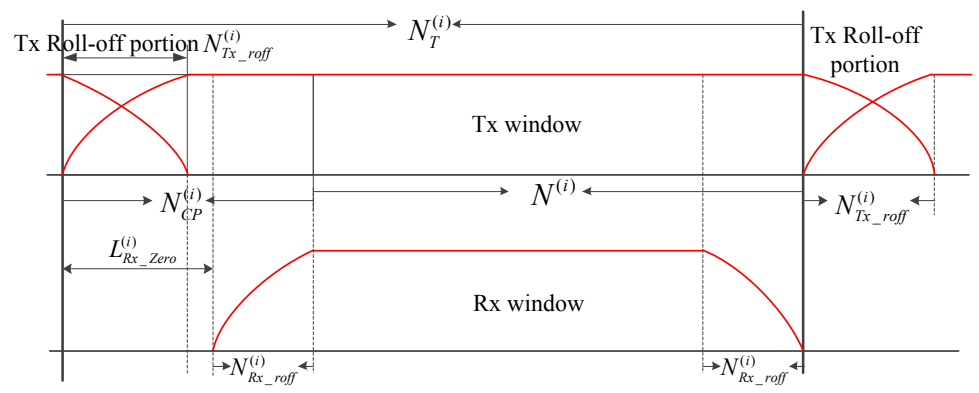

Fig. 1. The illustration of transmit and receive windows.

with the diagonal elements taken from the vector $\mathbf{x} . \mathbf{1}_{N}$ is a $N \times 1$ vector with all the element being $1 . \otimes$ represents Kronecker product. $\overline{g(t)}$ denotes the conjugate of the function $g(t)$. Blkdiag $(\mathbf{A}, N)$ is a block diagonal matrix generated by repeating A matrix $N$ times. $\lfloor a\rfloor$ denotes the largest integer smaller than or equal to $a$. For a matrix $\mathbf{A}, \mathbf{A}(i,:)$ and $\mathbf{A}(:, j)$ denote the $i$ th row and the $j$ th column of matrix $\mathbf{A}$, respectively. We use $i$ in parentheses, i.e., $(i)$ to denote the subband link using numerology $i$.

\section{SYSTEM DESCRIPTION WITH SINGLE NUMEROLOGY}

Let us first consider a single numerology W-OFDM system. Assume that there are $Z^{(i)}$ continuous subcarriers in a subband using numerology $i$. The discrete-time baseband W-OFDM signal is given by:

$$
s^{(i)}(n)=\sum_{u=0}^{Z^{(i)}-1} \sum_{v=-\infty}^{\infty} x_{u, v}^{(i)} g_{u, v}^{(i)}(n)
$$

where $x_{u, v}^{(i)}$ denotes the independent identically distributed (i.i.d) complex modulation symbol with zero mean and unit power on the $u$ th subcarrier of the $v$ th W-OFDM symbol. The synthesis function $g_{u, v}^{(i)}(n)$ for the $u$ th subcarrier of the $v$ th symbol is defined as

$g_{u, v}^{(i)}(n)=\frac{1}{\sqrt{N^{(i)}}} g_{u}^{(i)}\left(n-v N_{T}^{(i)}\right) e^{j \frac{2 \pi}{N(i)}\left(u+\Delta F^{(i)}\right)\left(n-N_{C P}^{(i)}-v N_{T}^{(i)}\right)}$,

where $N_{T}^{(i)}=N_{C P}^{(i)}+N^{(i)}$ denotes the symbol duration with CP length $N_{C P}^{(i)}$ and discrete Fourier transform (DFT) length $N^{(i)} . \Delta F^{(i)}$ denotes the frequency offset of the subband. As illustrated in Fig. 1, $g_{u}^{(i)}(n)$ for $0 \leq n<N_{w}^{(i)}$ is the transmit window with unit response in the middle $N_{T}^{(i)}-N_{\text {Tx_roff }}^{(i)}$ samples and smooth roll-off portions for the first and last $N_{\text {Tx_roff }}^{(i)}$ samples. For W-OFDM systems, the first $N_{\text {Tx_roff }}^{(i)}$ samples of $\mathrm{CP}$ and the last $N_{\text {Tx_roff }}^{(i)}$ samples of cyclic suffix (CS) are weighted by the roll-off portions of the window [12], which constitute the smooth ramp up and ramp down transitions to reduce the OOBE. To obtain the specific symbol rate as OFDM, the adjacent symbols overlap in the roll-off regions. When $N_{\text {Tx_roff }}^{(i)}=0$, the window becomes rectangular pulse with large sidelobes and the W-OFDM system degenerates to the OFDM system as a special case.
Let us assume that the discrete channel impulse response (CIR) for the subband (user) with the $i$ th numerology can be written as $\mathbf{h}^{(i)}=\left[h^{(i)}(0), \cdots, h^{(i)}\left(L_{C H}^{(i)}-1\right)\right]^{T}$, where $L_{C H}^{(i)}$ is the channel delay spread. Assume that the multipath channel is time invariant for the considered W-OFDM symsbols. The received signal is given by:

$$
y^{(i)}(n)=\sum_{l=0}^{L_{C H}^{(i)}-1} h^{(i)}(l) s^{(i)}(n-l)+\tilde{w}^{(i)}(n),
$$

where $\tilde{w}^{(i)}(n)$ is the additive white Gaussian noise (AWGN). The received signal is correlated with the analysis function and then sampled to recover the symbol. The demodulated symbol on the $k$ th subcarrier of the $m$ th symbol is obtained as

$$
\hat{y}_{k, m}^{(i)}=\sum_{n} y^{(i)}(n) \overline{q_{k, m}^{(i)}(n)},
$$

where the analysis function $q_{k, m}^{(i)}(n)$ is defined as

$q_{k, m}^{(i)}(n)=\frac{1}{\sqrt{N^{(i)}}} q_{k}^{(i)}\left(n-m N_{T}^{(i)}\right) e^{j \frac{2 \pi}{N^{(i)}}\left(k+\Delta F^{(i)}\right)\left(n-N_{C P}^{(i)}-m N_{T}^{(i)}\right)}$.

In (5), $q_{k}^{(i)}(n), 0 \leq n<N_{T}^{(i)}$, is the receive window for the $k$ th subcarrier using numerology $i$. As shown in Fig. 1, $q_{k}^{(i)}(n)$ has roll-off portions and unit response portion similar to the transmit window. To maintain the orthogonality, the first $L_{\mathrm{Rx} \_ \text {Zero }}^{(i)}$ elements of the receive window are set to be zeros, thus a sufficient condition to avoid ISI is

$$
L_{\text {Rx_Zero }}^{(i)}-N_{\text {Tx_roff }}^{(i)} \geq L_{C H}^{(i)}-1 .
$$

The length of roll-off part of the receive window is $N_{\mathrm{Rx} \_ \text {roff }}^{(i)}$ which satisfies $L_{\mathrm{Rx} \text { _Zero }}^{(i)}+N_{\mathrm{Rx} \text { roff }}^{(i)}=N_{C P}^{(i)}$. Consider the raised cosine (RC) window suggested by [23] for both transmitter and receiver windows, if (6) holds true and the receiver window has vestigial symmetry, i.e.,

$$
q_{k}^{(i)}(n)+q_{k}^{(i)}\left(n+N^{(i)}\right)=1,0 \leq n \leq N_{C P}^{(i)}-1,
$$

we have

$$
\frac{1}{N^{(i)}} \sum_{n=0}^{N_{T}^{(i)}-1} q_{k}^{(i)}(n) e^{-j \frac{2 \pi}{N^{(i)}}(k-u)\left(n-N_{C P}^{(i)}\right)}=\delta(k-u),
$$

where $\delta(\cdot)$ is Dirac delta function. Substituting (1) and (3) into (4), after some manipulation shown in Appendix A, (4) can 
be can be simplified as

$$
\hat{y}_{k, m}^{(i)}=x_{k, m}^{(i)} H^{(i)}(k)+w^{(i)}(k) .
$$

where $H^{(i)}(k)=\sum_{l=0}^{L_{C H}^{(i)}-1} h^{(i)}(l) e^{-j \frac{2 \pi}{N^{(i)}}\left(k+\Delta F^{(i)}\right)(l)}$ is the channel frequency response (CFR) on the $k$ th subcarrier of subband using numerology $i, w^{(i)}(k)$ is the corresponding AWGN on the $k$ th subcarrier with the variance $\sigma_{(i)}^{2}(k)$. We assume that the subchannel of each subcarrier is flat fading, and both (6) and (7) hold throughout the paper. Therefore, there are no ISI and intercarrier interference (ICI) in the underlying W-OFDM systems.

The modulation and demodulation process indicated by (1)(9) can be reformulated in vector/matrix notation. Let us assume that $N_{s}^{(i)}$ W-OFDM symbols are transmitted. The information symbols can be expressed in a $N_{s}^{(i)} Z^{(i)} \times 1$ vector $\mathbf{x}_{N_{s}^{(i)}}^{(i)}$ as

$$
\begin{aligned}
\mathbf{x}_{N_{s}^{(i)}}^{(i)}= & {\left[x_{0,0}^{(i)}, x_{1,0}^{(i)}, \cdots, x_{Z^{(i)}-1,0}^{(i)}, x_{0,1}^{(i)}, \cdots, x_{Z^{(i)}-1,1}^{(i)},\right.} \\
& \left.x_{0, N_{s}^{(i)}-1}^{(i)}, \cdots, x_{Z^{(i)}-1, N_{s}^{(i)}-1}^{(i)}\right]^{T} .
\end{aligned}
$$

The received signal $\mathbf{y}_{N_{s}^{(i)}}^{(i)} \in \mathbb{C}^{N_{s}^{(i)} N_{T}^{(i)} \times 1}$ can be written as

$$
\mathbf{y}_{N_{s}^{(i)}}^{(i)}=\Psi_{N_{s}^{(i)}}^{(i)} \mathbf{G}_{N_{s}^{(i)}}^{(i)} \mathbf{x}_{N_{s}^{(i)}}^{(i)}+\tilde{\mathbf{w}}^{(i)},
$$

with

$$
\begin{gathered}
\mathbf{G}_{N_{s}^{(i)}}^{(i)}=\left[\mathbf{g}_{0,0}^{(i)} \mathbf{g}_{1,0}^{(i)}, \cdots, \mathbf{g}_{Z^{(i)}-1,0}^{(i)}, \mathbf{g}_{0,1}^{(i)} \mathbf{g}_{1,1}^{(i)}, \cdots\right. \\
\left.\mathbf{g}_{0, N_{s}^{(i)}-1}^{(i)}, \cdots, \mathbf{g}_{Z^{(i)}-1, N_{s}^{(i)}-1}^{(i)}\right]
\end{gathered}
$$

where $\mathbf{G}_{N_{s}^{(i)}}^{(i)}$ is the $L^{(i)} \times N_{s}^{(i)} Z^{(i)}$ transmit matrix with $L^{(i)}=N_{s}^{(i)} N_{T}^{(i)}+N_{\text {Tx_roff }}^{(i)}$ being the total signal length. $\mathbf{g}_{u, v}^{(i)}$, for $0 \leq u<Z^{(i)}$ and $0 \leq v<N_{s}^{(i)}$, is a $L^{(i)} \times 1$ vector with the $n$th element $\left\{g_{u, v}^{(i)}(n)\right\}_{n=0}^{\left(L^{(i)}-1\right)}$. Since the last $N_{\text {Tx_roff }}^{(i)}$ CS samples are neglected, $\Psi_{N_{s}^{(i)}}^{(i)} \in \mathbb{C}^{N_{s}^{(i)} N_{T}^{(i)} \times L^{(i)}}$ is a submatrix constructed by the first $N_{s}^{(i)} N_{T}^{(i)}$ rows of the $L^{(i)}$-dimensional Toeplitz channel square matrix whose first column and first row are $\left[h^{(i)}(0), \cdots, h^{(i)}\left(L_{C H}^{(i)}-1\right) \mathbf{0}_{1 \times\left(N_{s}^{(i)} N_{T}^{(i)}-L_{C H}^{(i)}\right)}\right]^{T} \in$ $\mathbb{C}^{N_{s}^{(i)} N_{T}^{(i)} \times 1}$ and $\left[h^{(i)}(0), \mathbf{0}_{1 \times\left(L^{(i)}-1\right)}\right] \in \mathbb{C}^{1 \times L^{(i)}}$, respectively. The demodulated signal can be written as

$$
\hat{\mathbf{y}}_{N_{s}^{(i)}}^{(i)}=\mathbf{Q}_{N_{s}^{(i)}}^{(i)} \mathbf{y}_{N_{s}^{(i)}}^{(i)}+\mathbf{w}^{(i)}
$$

where $\hat{\mathbf{y}}_{N_{s}^{(i)}}^{(i)}=\left[\hat{y}_{0,0}^{(i)}, \hat{y}_{1,0}^{(i)}, \cdots, \hat{y}_{Z^{(i)}-1,0}^{(i)}, \hat{y}_{0,1}^{(i)}, \cdots, \hat{y}_{Z^{(i)}-1,1}^{(i)}\right.$, $\left.\hat{y}_{0, N_{s}^{(i)}-1}^{(i)}, \cdots, \hat{y}_{Z^{(i)}-1, N_{s}^{(i)}-1}^{(i)}\right]^{T}$ is a $N_{s}^{(i)} Z^{(i)} \times 1$ demodulated signal and $\mathbf{w}^{(i)} \in \mathbb{C}^{N_{s}^{(i)} Z^{(i)} \times 1}$ is the corresponding Gaussian noise vector. The receive window $\mathbf{Q}_{N_{s}^{(i)}}^{(i)} \in \mathbb{C}^{N_{s}^{(i)} Z^{(i)} \times N_{s}^{(i)} N_{T}^{(i)}}$ is defined by

$$
\begin{gathered}
\mathbf{Q}_{N_{s}^{(i)}}^{(i)}=\left[\mathbf{q}_{0,0}^{(i)}, \mathbf{q}_{1,0}^{(i)}, \cdots, \mathbf{q}_{Z^{(i)}-1,0}^{(i)}, \mathbf{q}_{0,1}^{(i)}, \mathbf{q}_{1,1}^{(i)}, \cdots,\right. \\
\left.\mathbf{q}_{0, N_{s}^{(i)}-1}^{(i)}, \cdots, \mathbf{q}_{Z^{(i)}-1, N_{s}^{(i)}-1}^{(i)}\right]^{T}
\end{gathered}
$$

where $\mathbf{q}_{k, m}^{(i)} \in \mathbb{C}^{\left(N_{s}^{(i)} N_{T}^{(i)}\right) \times 1}$, for $0 \leq k<Z^{(i)}$ and $0 \leq m<$ $N_{s}^{(i)}$, is the demodulation vector with the $n$th the element $\left\{q_{k, m}^{(i)}(n)\right\}_{n=0}^{\left(N_{s}^{(i)} N_{T}^{(i)}-1\right)}$. According to (9), (13) can be simplified

$$
\hat{\mathbf{y}}_{N_{s}^{(i)}}^{(i)}=\mathbf{H}_{N_{s}^{(i)}}^{(i)} \mathbf{x}_{N_{s}^{(i)}}^{(i)}+\mathbf{w}^{(i)},
$$

where $\quad \mathbf{H}_{N_{s}^{(i)}}^{(i)}=\operatorname{Blkdiag}\left(\mathbf{H}^{(i)}, N_{s}^{(i)}\right)$ with $\mathbf{H}^{(i)}=$ $\operatorname{diag}\left\{\left[H^{(i)}(0), \cdots, H^{(i)}\left(Z^{(i)}-1\right)\right]^{T}\right\} \quad$ is $\quad$ a $\quad N_{s}^{(i)} Z^{(i)}$ dimensional CFR matrix. The variance matrix of noise vector $\mathbf{w}^{(i)}$ is $\Gamma_{N_{s}^{(i)}}^{(i)}=\operatorname{Blkdiag}\left(\boldsymbol{\Gamma}^{(i)}, N_{s}^{(i)}\right)$ with $\boldsymbol{\Gamma}^{(i)}=$ $\operatorname{diag}\left\{\left[\sigma_{(i)}^{2}(0), \cdots, \sigma_{(i)}^{2}\left(Z^{(i)}-1\right)\right]^{T}\right\}$.

\section{SYSTEM MODEL FOR MIXED NUMEROLOGIES TRANSMISSION}

In the scenario of mixed numerologies transmission, the system bandwidth is divided into several subbands, each band is assigned one type of numerology. Considering a commonly accepted family of numerologies, the subcarrier spacing and CP length are defined as follows [7], [8], [27]:

$$
\Delta f^{(i)}=n_{i} \Delta f^{(i-1)}, N_{C P}^{(i)}=N_{C P}^{(i-1)} / n_{i}, n_{i}=2^{\kappa},
$$

where $\Delta f^{(i)}$ is the subcarrier spacing for the $i$ th numerology and $\kappa$ is an integer. We assume that different numerologies have the same sampling rate. Without loss of generality, we consider two adjacent subbands using two different numerologies with the GB $F_{G B}$, which are depicted in Fig. 2. Specifically, for two subbands, we have $\Delta f^{(2)}=n_{2} \Delta f^{(1)}$,

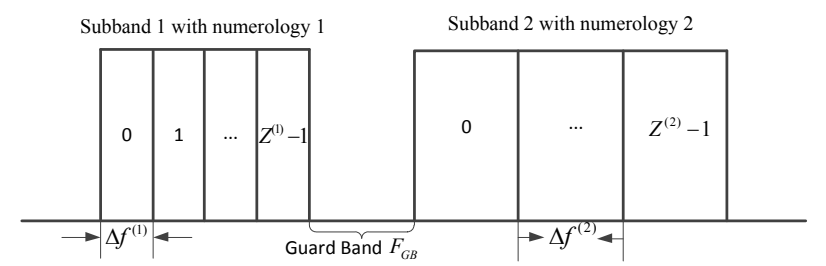

Fig. 2. An example of mixed numerologies transmission with two subbands.

$N_{C P}^{(1)}=n_{2} N_{C P}^{(2)}$. Let us consider the generalized synchronized scenario, an integral least common multiplier (LCM) symbol consists of $N_{T}^{(1)}$ samples [5], the subframe structures of different numerologies are aligned, and every $n_{2}$ symbols using numerology 2 have the same duration with that of one symbol using numerology 1 , i.e.,

$$
N_{T}^{(1)}=N^{(1)}+N_{C P}^{(1)}=n_{2}\left(N^{(2)}+N_{C P}^{(2)}\right)=n_{2} N_{T}^{(2)} .
$$

The transmitted signals from different subbands are added together before being sent to demodulator. Using (3), the received signal can be written as:

$$
\begin{aligned}
y(n)= & y^{(1)}(n)+y^{(2)}(n) \\
= & \rho^{(1)} \sum_{l=0}^{L_{C H}^{(1)}-1} h^{(1)}(l) s^{(1)}(n-l) \\
& \quad+\rho^{(2)} \sum_{l=0}^{L_{C H}^{(2)}-1} h^{(2)}(l) s^{(2)}(n-l)+\tilde{w}(n),
\end{aligned}
$$


where $\tilde{w}(n)$ denotes the AWGN term associated to $y(n), \rho^{(i)}$ denotes the power adjusting factor for the $i$ th subband. If the two subbands signal have the same transmit power then we have

$$
\rho^{(i)}=\sqrt{\frac{N^{(i)} L^{(i)}}{N_{w}^{(i)} N_{s}^{(i)} \sum_{u=0}^{Z(i)}-1} P_{g_{u}}^{(i)}},
$$

where $P_{g_{u}}^{(i)}$ denotes the average power of the transmit window $g_{u}^{(i)}(n)$. For the arbitrary subband using numerology $i$, the subchannel of each subcarrier is assumed to be flat fading in the considered use case scenario. Using (1)-(9), when $y(n)$ is demodulated by the receiver with numerology $i$, the demodulated signal on the $k$ th subcarrier of the $m$ th W-OFDM symbol can be written as

$$
\begin{aligned}
\hat{y}_{k, m}^{(i)}= & \sum_{n} y(n) \overline{q_{k, m}^{(i)}(n)} \\
= & \rho^{(i)} x_{k, m}^{(i)} H^{(i)}(k)+\rho^{(j)} \sum_{u=0}^{Z^{(j)}-1} \sum_{v=-\infty}^{\infty} x_{u, v}^{(j)} \sum_{l=0}^{L_{C H}^{(j)}-1} h^{(j)}(l) \\
& \times \sum_{n} g_{u, v}^{(j)}(n-l) \overline{q_{k, m}^{(i)}(n)}+w^{(i)}(k),
\end{aligned}
$$

where the first term is the desired signal, the second term is the INI caused by the subband using numerology $j, j \neq i$, the last term is Gaussian noise.

In order to simplify derivation for the INI cancellation algorithm which will be discussed in Section V, we neglect the overlapping of the adjacent LCM symbols and consider the system model for one LCM symbol in vector/matrix form. Set $N_{s}^{(1)}=1, N_{s}^{(2)}=n_{2}$ and use (11), the mixed numerologies received signal for one LCM symbol can be written as

$$
\mathbf{y}=\rho^{(1)} \mathbf{\Psi}_{1}^{(1)} \mathbf{G}_{1}^{(1)} \mathbf{x}_{1}^{(1)}+\rho^{(2)} \mathbf{\Psi}_{n_{2}}^{(2)} \mathbf{G}_{n_{2}}^{(2)} \mathbf{x}_{n_{2}}^{(2)}+\mathbf{w}
$$

where $\mathbf{y}=\left[y(0), \cdots, y\left(N_{T}^{(1)}\right)\right]^{T}$ is the compound received signal from both subbands. $\Psi_{1}^{(1)} \in \mathbb{C}^{N_{T}^{(1)} \times L^{(1)}}, \mathbf{G}_{1}^{(1)} \in$ $\mathbb{C}^{L^{(1)} \times Z^{(1)}}, \mathbf{x}_{1}^{(1)} \in \mathbb{C}^{Z^{(1)} \times 1}$ and $\mathbf{\Psi}_{n_{2}}^{(2)} \in \mathbb{C}^{n_{2} N_{T}^{(2)} \times L^{(2)}}, \mathbf{G}_{n_{2}}^{(2)} \in$ $\mathbb{C}^{L^{(2)} \times n_{2} Z^{(2)}}, \mathbf{x}_{n_{2}}^{(2)} \in \mathbb{C}^{n_{2} Z^{(2)} \times 1}$ are the channel matrices, transmit matrices and information symbols of subband 1 and subband 2 for one LCM symbol duration, respectively. $\mathbf{w} \in \mathbb{C}^{N_{T}^{(1)} \times 1}$ is the AWGN noise. According to (13), (14) and (15), the demodulated signal obtained by the receiver using numerology 1 is given as

$$
\hat{\mathbf{y}}_{1}^{(1)}=\mathbf{Q}_{1}^{(1)} \mathbf{y}=\rho^{(1)} \mathbf{H}_{1}^{(1)} \mathbf{x}_{1}^{(1)}+\mathbf{E}_{(1,2)} \mathbf{x}_{n_{2}}^{(2)}+\mathbf{w}^{(1)},
$$

where $\mathbf{H}_{1}^{(1)} \in \mathbb{C}^{Z^{(1)} \times Z^{(1)}}$ is the diagonal CFR matrix for one W-OFDM symbol of subband 1. $\mathbf{E}_{(1,2)}=\rho^{(2)} \mathbf{Q}_{1}^{(1)} \mathbf{\Psi}_{n_{2}}^{(2)} \mathbf{G}_{n_{2}}^{(2)}$ is the $Z^{(1)} \times n_{2} Z^{(2)}$ interference matrix and $\mathbf{E}_{(1,2)} \mathbf{x}_{n_{2}}^{(2)}$ denotes the INI of subband 1 suffering from the adjacent subband using numerology 2. $\rho^{(1)} \mathbf{H}_{1}^{(1)} \mathbf{x}_{1}^{(1)}$ in (22) is the desired signal term. $\mathbf{w}^{(1)}$ is the Gaussian noise vector whose variance vector is $\gamma^{(1)}=\left[\sigma_{(1)}^{2}(0), \cdots, \sigma_{(1)}^{2}\left(Z^{(1)}-1\right)\right]^{T}$. Similarly, the demodulated signal $\hat{\mathbf{y}}_{n_{2}}^{(2)} \in \mathbb{C}^{n_{2} Z^{(2)} \times 1}$ using numerology 2 can be written as

$$
\hat{\mathbf{y}}_{n_{2}}^{(2)}=\mathbf{Q}_{n_{2}}^{(2)} \mathbf{y}=\rho^{(2)} \mathbf{H}_{n_{2}}^{(2)} \mathbf{x}_{n_{2}}^{(2)}+\mathbf{E}_{(2,1)} \mathbf{x}_{1}^{(1)}+\mathbf{w}^{(2)},
$$

where $\mathbf{H}_{n_{2}}^{(2)}=\operatorname{BlkDiag}\left(\mathbf{H}_{1}^{(2)}, n_{2}\right)$ is the CFR matrix for $n_{2}$ W-OFDM symbols of subband using numerology 2 , where $\mathbf{H}_{1}^{(2)}=\operatorname{diag}\left\{\left[H^{(2)}(0), \cdots, H^{(2)}\left(Z^{(2)}-1\right)\right]^{T}\right\} . \quad \mathbf{E}_{(2,1)}=$ $\rho^{(1)} \mathbf{Q}_{n_{2}}^{(2)} \mathbf{\Psi}_{1}^{(1)} \mathbf{G}_{1}^{(1)}$ is the $n_{2} Z^{(2)} \times Z^{(1)}$ interference matrix of subband 2 and $\mathbf{E}_{(2,1)} \mathbf{x}_{1}^{(1)}$ denotes the INI of subband 2 suffering from subband 1 . The variance of noise vector $\mathbf{w}^{(2)}$ is $\gamma_{n_{2}}^{(2)}=\mathbf{1}_{n_{2}} \otimes \gamma^{(2)}$ with $\boldsymbol{\gamma}^{(2)}=\left[\sigma_{(2)}^{2}(0), \cdots, \sigma_{(2)}^{2}\left(Z^{(2)}-1\right)\right]^{T}$. Next we shall first analyze the power of INI for both subbands, and then propose the interference cancellation algorithm.

\section{INTER-NUMEROLOGY INTERFERENCE ANALYSIS}

In this section, we discuss the INI for two adjacent subbands using different numerologies under multipath fading channels.

\section{A. Inter-Numerology Interference Analysis for Numerology 1}

First, we consider the subband using numerology 1 which is with small subcarrier spacing $\Delta f^{(1)}$. This subband suffers from the INI caused by subband 2. Using (20) and set $i=$ $1, j=2$, the INI suffered by the $k$ th subcarrier of the $m$ th W-OFDM symbol in this subband can be written as:

$$
I^{(1)}(k, m)=\sum_{u=0}^{Z^{(2)}-1} I_{u}^{(1)}(k, m),
$$

with

$I_{u}^{(1)}(k, m)=\rho^{(2)} \sum_{v=-\infty}^{\infty} x_{u, v}^{(2)} \sum_{l=0}^{L_{C H}^{(2)}-1} h^{(2)}(l) \sum_{n} g_{u, v}^{(2)}(n-l) \overline{q_{k, m}^{(1)}(n)}$,

where $I_{u}^{(1)}(k, m)$ is the component INI of $I^{(1)}(k, m)$ caused by the $u$ th subcarrier of subband 2 . Since the transmit signal is i.i.d, the power of $I_{u}^{(1)}(k, m)$ can be expressed as:

$$
P_{u}^{(1)}(k, m)=\sum_{v=-\infty}^{\infty}\left|\rho^{(2)} \sum_{l=0}^{L_{C H}^{(2)}-1} h^{(2)}(l) \sum_{n} g_{u, v}^{(2)}(n-l) \overline{q_{k, m}^{(1)}(n)}\right|^{2} .
$$

The total interference power at the $k$ th subcarrier of the $m$ th symbol can be written as:

$$
P^{(1)}(k, m)=\sum_{u=0}^{Z^{(2)}-1} P_{u}^{(1)}(k, m) .
$$

Without loss of generality, we set $\Delta F^{(1)}=0$ for subband 1 . By substituting (2) and (5) into (26) and assuming the channel delay spread is small compared to the symbol duration, we have

$$
\begin{aligned}
P_{u}^{(1)}(k, m) \approx & \left.\frac{\rho^{(2)}}{\sqrt{N^{(1)} N^{(2)}}}\right)^{2}\left|H^{(2)}(u)\right|^{2} \\
& \times \sum_{d=-1}^{n_{2}-1}\left|\sum_{n} \Omega_{\substack{q_{k}^{(1)}, 0 \\
g_{u}^{(2)}, d N_{T}^{(2)}}}^{(n)} e^{-j \frac{2 \pi}{N^{(1)}} \Delta k^{(1)} n}\right|^{2} .
\end{aligned}
$$




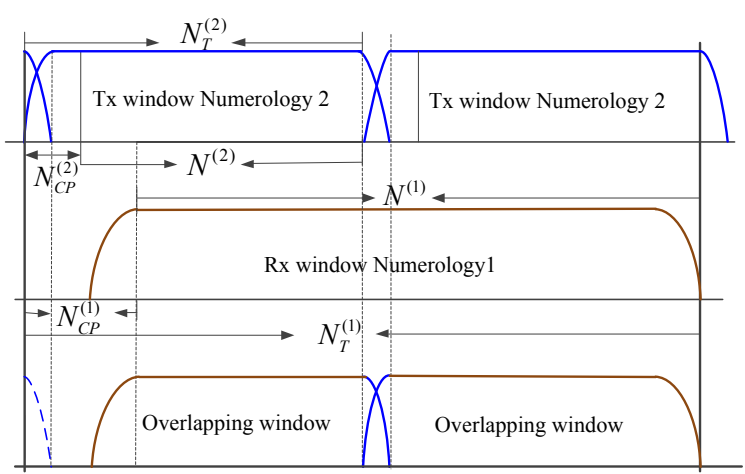

Fig. 3. INI analysis for subband using numerology 1.

Detailed derivations for (28) can be found in Appendix B. In (28), we use $\Omega_{g, \zeta}^{q, \tau}(n) \triangleq q(n-\tau) g(n-\zeta)$ to denote the overlapping window generated by the time-shifted function $g(n-\zeta)$ and $q(n-\tau) \cdot \Delta k^{(1)}=k-\hat{k}(u)$ is the spectral distance between the victim subcarrier $k$ of subband 1 and the interfering subcarrier $\hat{k}(u)$ of subband 2 , where $\hat{k}(u)=$ $n_{2}\left(u+\Delta F^{(2)}\right)$, for $0 \leq u<Z^{(2)}$, represents the subcarrier indexes of subband 2 when the subcarriers are observed with the granularity of numerology 1 , i.e., subcarrier spacing $\Delta f^{(1)}$. $H^{(2)}(u)=\sum_{l=0}^{L_{C H}^{(2)}-1} h^{(2)}(l) e^{-j \frac{2 \pi}{N^{(2)}}\left(u+\Delta F^{(2)}\right) l}$ is the CFR on the $u$ th subcarrier of subband 2. From (28), one can see that the INI power is a function of the CFR of interfering subband, the spectral distance of the victim subcarrier and the interfering subcarrier, and the DFT magnitude of the overlapping window $\Omega_{g_{u}^{(2)}, d N_{T}^{(2)}}^{q^{(1)}, 0}(n)$ which is generated by the time-shifted transmit windows of the aggressor subcarrier $g_{u}^{(2)}\left(n-d N_{T}^{(2)}\right)$ and the receive window of the victim subcarrier $q_{k}^{(1)}(n)$. The overlapping windows in the scenario of mixed numerologies transmission with $n_{2}=2$ are illustrated in Fig. 3 .

As can be observed from Fig. 3 and (28), there are $n_{2}+1$ transmit windows that may possibly overlap with the receiver window of numerology 1 . Herein $g_{u}^{(2)}\left[n-\left(n_{2} m-1\right) N_{T}^{(2)}\right]$, i.e., the transmit window of the previous LCM symbol for subband 2 , is also taken into account due to the overlapping of the adjacent transmit windows. Note that, in some system parameters settings, it may not overlap with the considered receive window of numerology 1, and thus would not contribute to the INI of numerology 1 . See the dashed curve in overlapping window of Fig. 3. In Fig. 3, all the overlapping windows have smooth transition edges which come from the roll-off portions of the victim's receiver window or the aggressor' transmit windows. When the two subbands use the same numerology, the overlapping window becomes the receiver window of numerology 1 , whose $N^{(1)}$-point DFT satisfies (8). Therefore in this case there are no INI. As indicated by (28), under the condition of generalized synchronization, the power of INI for numerology 1 is not related to the symbol index $m$, thus $P_{u}^{(1)}(k)$ is used instead of $P_{u}^{(1)}(k, m)$ in the rest of the paper.

As a special case of W-OFDM, the INI for CP-OFDM systems can also be derived based on (28). In this case, $g_{u}^{(2)}(n)$

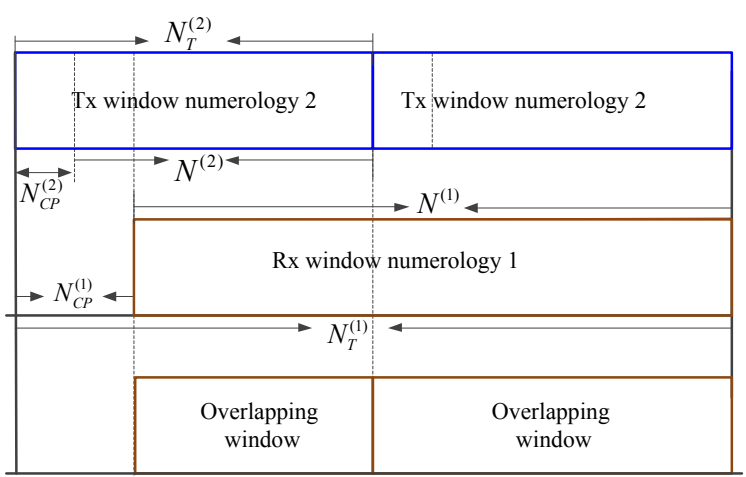

Fig. 4. INI analysis for numerology 1 in CP-OFDM case.

and $q_{k}^{(1)}(n)$ are rectangular windows with the length $N_{T}^{(2)}$ and $N^{(1)}$, respectively, which lead to rectangular overlapping windows, as depicted in Fig. 4. The INI power $\tilde{P}_{u}^{(1)}(k)$ caused by the $u$ th subcarrier of subband 2 for the $k$ th subcarrier of subband 1 is given by

$$
\begin{gathered}
\breve{P}_{u}^{(1)}(k) \approx \frac{\left|\rho^{(2)} H^{(2)}(u)\right|^{2}}{N^{(2)} N^{(1)}}\left[\left|\frac{\sin \left[\frac{\pi}{N^{(1)}} \Delta k^{(1)} \alpha N_{T}^{(2)}\right]}{\sin \left[\frac{\pi}{N^{(1)}} \Delta k^{(1)}\right]}\right|^{2}\right. \\
\left.+\left.\alpha\left|\frac{\sin \left[\frac{\pi}{N^{(1)}} \Delta k^{(1)} N_{T}^{(2)}\right]}{\sin \left[\frac{\pi}{N^{(1)}} \Delta k^{(1)}\right]}\right|\right|^{2}\right],
\end{gathered}
$$

where $\alpha=\left\lfloor N^{(1)} / N_{T}^{(2)}\right\rfloor$ is the number of rectangular overlapping windows with length $N_{T}^{(2)}$. The detailed derivation of (29) is given in Appendix C. When the two subbands have the same numerology, then $\alpha=0$ and the power of INI is zero.

From (29) and (28), we can see the difference between the CP-OFDM and W-OFDM is that the overlapping windows of $\mathrm{CP}-\mathrm{OFDM}$ are all rectangular windows with sharp transitions, while for W-OFDM the overlapping windows have smooth transition edges which are inherited from the transmit windows of the interfering subcarrier and the receive window of victim subcarrier. Since the INI power depends on the DFT magnitude of overlapping windows at the spectral distance $\Delta k^{(1)}$, the smooth transitions of the overlapping windows in time domain may lead to faster decaying spectrum magnitude and hence reduce the INI expanding. From this point, we can intuitively understand that both the transmit window and the receiver window of W-OFDM would affect the INI level.

\section{B. Inter-Numerology interference Analysis for Numerology 2}

The INI suffered by subband using numerology 2 is discussed in this section. Similar to the derivation of (26), the INI power caused by the $u$ th subcarrier of subband 1 on the $k$ th subcarrier of the $m$ th symbol for the subband 2 can be expressed as:

$P_{u}^{(2)}(k, m)=\sum_{v=-\infty}^{\infty}\left|\rho^{(1)} \sum_{l=0}^{L_{C H}^{(1)}-1} h^{(1)}(l) \sum_{n} g_{u, v}^{(1)}(n-l) \overline{q_{k, m}^{(2)}(n)}\right|^{2}$. 


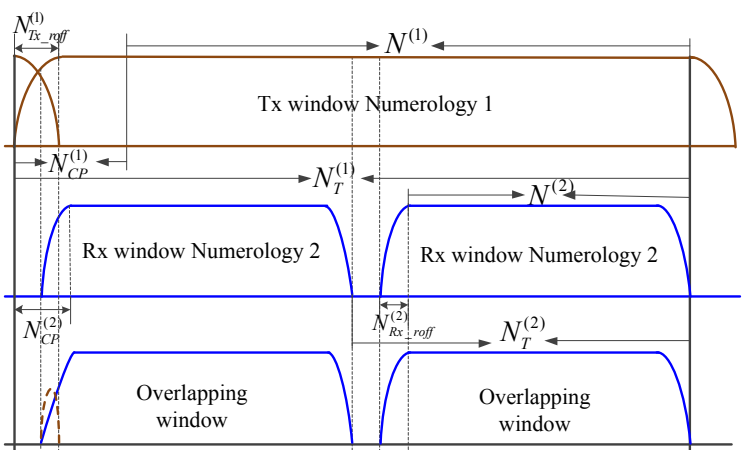

Fig. 5. INI analysis for subband using numerology 2.

We consider one LCM symbol consisting of $N_{T}^{(1)}$ samples in the generalized synchronous scenario [5]. Assume that the $m$ th received symbol of subband 2 belongs to the $\tilde{m}$ th LCM symbol, therefore it may be interfered by the $\tilde{m}$ and $(\tilde{m}-1)$ th transmitted symbol of subband 1 , where $\tilde{m}=\left\lfloor\frac{m}{n_{2}}\right\rfloor$. We set $m=\tilde{m} n_{2}+d$ with $d=0,1, \cdots, n_{2}-1$, where $d$ denotes the relative position index for the $m$ th receive window of subband 2 within one LCM symbol. We assume the channel is weakly or mildly time-dispersive and thus the channel delay spread is small compared to the symbol duration. Substituting (2) and (5) into (30) and after some manipulations shown in Appendix $\mathrm{D}$, the power of component INI can be written as

$$
\begin{aligned}
P_{u}^{(2)}(k, m) \approx & \left.\frac{\rho^{(1)}}{\sqrt{N^{(2)} N^{(1)}}}\right)^{2}\left|H^{(1)}(u)\right|^{2} \\
& \times \sum_{j=0}^{1}\left|\sum_{n} \Omega_{g_{u}^{(1)},-j N_{T}^{(1)}}^{q^{(2)},(2)}(n) e^{-j \frac{2 \pi}{N^{(1)}} \Delta k^{(2)} n}\right|^{2} .
\end{aligned}
$$

In (31), $H^{(1)}(u)=\sum_{l=0}^{L_{C H}^{(1)}-1} h^{(1)}(l) e^{-j \frac{2 \pi}{N(1)} u l}$ is the CFR on the $u$ th subcarrier of subband $1, \Delta k^{(2)}=\hat{k}(k)-u$ represents the spectral distance between the $k$ th subcarrier of subband 2 and the $u$ th subcarrier of subband 1 , where $\hat{k}(k)=n_{2}\left(k+\Delta F^{(2)}\right)$ denotes the subcarrier indexes of subband 2 when its $k$ th subcarrier is observed with the subcarrier spacing of numerology 1. $\Omega_{g_{u}^{(1)},-j N_{T}^{(1)}}^{q_{k}^{(2)}}, d N_{T}^{(2)}(n)$ is the overlapping window generated by $g_{u}^{(1)}\left(n+j N_{T}^{(1)}\right)$ and $q_{k}^{(2)}\left(n-d N_{T}^{(2)}\right)$, which is illustrated in Fig. 5. Note that the interference from the $(\tilde{m}-1)$ th transmitted symbol of subband 1 is also considered, which is shown as the dashed overlapping part in Fig. 5. Similarly, from (31), we can see that the power of INI depends on the CFR of the interfering subcarrier from subband 1 and the DFT magnitudes of overlapping windows at the subcarrier distance $\Delta k^{(2)}=\hat{k}(k)-u$. As shown in Fig. 5, during one LCM symbol, the first received signal of the subband 2 may be interfered by the previous LCM symbol of subband 1 due to the overlapping of roll-off portions. This may induce slight difference to the INI power for different receive windows in one LCM symbol duration for subband 2. Therefore, we define the average power of INI to measure the INI suffered by the

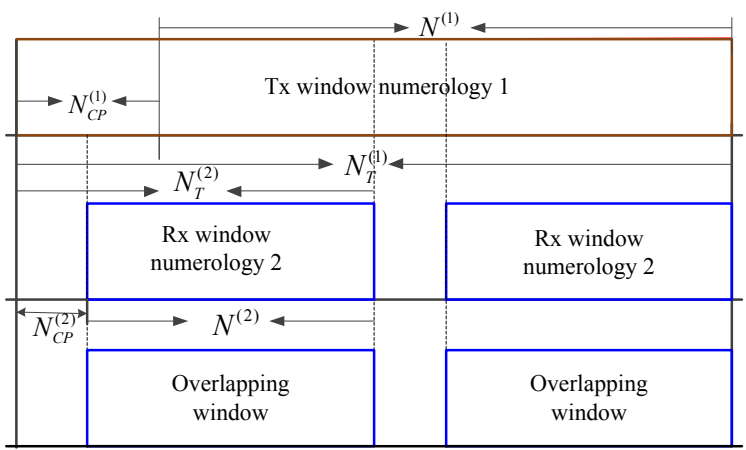

Fig. 6. INI analysis for numerology 2 in CP-OFDM case.

$k$ th subcarrier of subband 2 as follows

$$
P_{u}^{(2)}(k)=\frac{1}{n_{2}} \sum_{m=\tilde{m} n_{2}}^{\tilde{m} n_{2}+n_{2}-1} P_{u}^{(2)}(k, m) .
$$

As a special case of W-OFDM, the INI power for OFDM systems can be derived based on (31). The INI power caused by the $u$ th subcarrier of subband 1 on the $k$ th subcarrier using numerology 2 can be written as

$$
\breve{P}_{u}^{(2)}(k) \approx \frac{\left|\rho^{(1)} H^{(1)}(u)\right|^{2}}{N^{(2)} N^{(1)}}\left|\frac{\sin \left(\frac{\pi}{N^{(1)}} \Delta k^{(2)} N^{(2)}\right)}{\sin \left(\frac{\pi}{N^{(1)}} \Delta k^{(2)}\right)}\right|^{2} .
$$

As illustrated in Fig. 6, we can find that the overlapping windows are all rectangular with length $N^{(2)}$ in the case of OFDM, which have relatively large and slow decreasing spectral sidelobes. While the overlapping windows of WOFDM shown in Fig. 5 always have smooth transitions at both edges, which is mostly due to the roll-off parts of the receive window of the victim subcarrier. These smooth transitions reduce the expanding of INI compared with the rectangular overlapping windows. The quantitative comparison of SIRs for both subbands between OFDM and W-OFDM will be shown in Fig. 8 of Section VI.

With (28) and (32), for a given victim subcarrier $k$ using numerology $i$, the component SIR caused by a specific interfering subcarrier $u$ using numerology $j$ can be written as

$$
R_{u}^{(i)}(k)=\frac{P_{D}^{(i)}(k)}{P_{u}^{(i)}(k)}, 0 \leq k<Z^{(i)}, 0 \leq u<Z^{(j)},
$$

where $P_{D}^{(i)}(k)=\left|\rho^{(i)} H^{(i)}(k)\right|^{2}$ is the power of desired signal based on (20). The total SIR can be given by the following expression

$$
R_{T}^{(i)}(k)=\frac{P_{D}^{(i)}(k)}{P_{I}^{(i)}(k)}, 0 \leq k<Z^{(i)},
$$

where $P_{I}^{(i)}(k)=\sum_{u=0}^{Z^{(j)}-1} P_{u}^{(i)}(k)$ is the total power of INI from the adjacent subband using numerology $j$.

\section{INTER-NUMEROLOGY INTERFERENCE CANCELLATION}

In this section, we describe the proposed INI cancellation algorithm. With the aid of the analytical results of INI power 
from Section IV, it can be found that the INI power suffered and imposed by different subcarriers are diverse. As shown in Fig. 7, the edge subcarriers may cause and undergo relatively serious INI, while the INI power for the subband 1 with small subcarrier spacing show some fluctuations. Based on the analytical results of the INI power, all the subcarriers can be divided into two sets according to the level of INI power: the first set includes those subcarriers who suffer from or impose dominant INI. The second set is the complement of the first one, which represent those subcarriers less affected by the INI. For the subcarriers belonging to the first set, a soft-output OSIC algorithm is proposed to mitigate the interference, while for subcarriers belonging to the second set, the interference is marginal and can be regarded as effective noise whose variances are taken into account for the calculation of the LLRs of bits. In this paper, we assume that the transmitted symbols are equiprobable for all the possible constellation symbols.

According to the affordable complexity of the receiver, the major victim subcarriers using numerology $i$ contaminated by severe INI are chosen by an SIR threshold $\mathcal{T}_{1}$ as follows

$$
\mathcal{A}_{v}^{(i)}=\left\{k \mid R_{T}^{(i)}(k)<\mathcal{T}_{1}, k \in\left[0, \cdots, Z^{(i)}-1\right]\right\},
$$

where $\mathcal{A}_{v}^{(i)}$ denotes the major victim subcarriers set of subband $i$. Those subcarriers with numerology $j, j \neq i$, which impose dominant interference to the victim subcarrier $k, k \in \mathcal{A}_{v}^{(i)}$, can be identified by finding $R_{u}^{(i)}(k)$ larger than a predefined SIR threshold $\mathcal{T}_{2}$ as follows

$\mathcal{A}_{a}^{(j)}=\left\{u \mid R_{u}^{(i)}(k)<\mathcal{T}_{2}, k \in \mathcal{A}_{v}^{(i)}, u \in\left[0, \cdots, Z^{(j)}-1\right]\right\}$,

where $\mathcal{A}_{a}^{(j)}$ denotes the dominant aggressor subcarrier$\mathrm{s}$ set of subband $j$. For subcarriers belong to $\mathcal{A}^{(i)}=$ $\mathcal{A}_{v}^{(i)} \cup \mathcal{A}_{a}^{(i)}, i=1,2$, a joint OSIC scheme is proposed based on the system model given by (21)-(23) for one L$\mathrm{CM}$ symbol. Assume there are $N_{\mathcal{A}}^{(1)}$ subcarriers in $\mathcal{A}^{(1)}=$ $\left\{k_{\mathcal{A}, 0}^{(1)}, k_{\mathcal{A}, 1}^{(1)}, \cdots, k_{\mathcal{A}, N_{\mathcal{A}}^{(1)}-1}^{(1)}\right\}$ and $N_{\mathcal{A}}^{(2)}$ subcarriers in $\mathcal{A}^{(2)}=$ $\left\{k_{\mathcal{A}, 0}^{(2)}, k_{\mathcal{A}, 1}^{(2)}, \cdots, k_{\mathcal{A}, N_{\mathcal{A}}^{(2)}-1}^{(2)}\right\}$. Since there are $n_{2}$ numerology 2 symbols in one LCM symbol, the subcarriers belonging to $\mathcal{A}^{(2)}$ correspond to elements with the indexes $\overrightarrow{\mathcal{A}}^{(2)}=\left\{k_{\mathcal{A}, 0}^{(2)}, \cdots, k_{\mathcal{A}, N_{\mathcal{A}}^{(2)}-1}^{(2)}, Z^{(2)}+k_{\mathcal{A}, 0}^{(2)}, \cdots, Z^{(2)}+\right.$ $\left.k_{\mathcal{A}, N_{\mathcal{A}}^{(2)}-1}^{(2)},\left(n_{2}-1\right) Z^{(2)}+k_{\mathcal{A}, 0}^{(2)}, \cdots,\left(n_{2}-1\right) Z^{(2)}+k_{\mathcal{A}, N_{\mathcal{A}}^{(2)}-1}^{(2)}\right\}$ of the LCM symbol $\mathbf{x}_{n_{2}}^{(2)}$. Using $\mathcal{A}^{(1)}$ and $\overrightarrow{\mathcal{A}}^{(2)}$, we can define the received signal $\tilde{\mathbf{y}}=\left[\left(\tilde{\mathbf{y}}_{\mathcal{A}^{(1)}}^{(1)}\right)^{T}\left(\tilde{\mathbf{y}}_{\overrightarrow{\mathcal{A}}^{(2)}}^{(2)}\right)^{T}\right]^{T}$ and the transmitted signal $\tilde{\mathbf{x}}=\left[\left(\mathbf{x}_{\mathcal{A}^{(1)}}^{(1)}\right)^{T}\left(\mathbf{x}_{\mathcal{A}^{(2)}}^{(2)}\right)^{T}\right]^{T}$ for the dominant aggressor and victim subcarriers, respectively. For $\tilde{\mathbf{y}}$ with length $\tilde{L}=N_{\mathcal{A}}^{(1)}+n_{2} N_{\mathcal{A}}^{(2)}, \tilde{\mathbf{y}}_{\mathcal{A}^{(1)}}^{(1)}$ is constructed by taking the entries with row indices belonging to $\mathcal{A}^{(1)}$ from $\hat{\mathbf{y}}_{1}^{(1)}, \tilde{\mathbf{y}}_{\overrightarrow{\mathcal{A}}^{(2)}}^{(2)}$ is formed by taking the entries with rows indices belonging to $\overrightarrow{\mathcal{A}}^{(2)}$ from $\hat{\mathbf{y}}_{n_{2}}^{(2)}$. Accordingly, for $\tilde{\mathbf{x}} \in \mathbb{C}^{\tilde{L} \times 1}$, $\mathbf{x}_{\mathcal{A}^{(1)}}^{(1)}$ is formed by taking the entries of $\mathbf{x}_{1}^{(1)}$ with indices belonging to $\mathcal{A}^{(1)} \cdot \mathbf{x}_{\overrightarrow{\mathcal{A}}^{(2)}}^{(2)}$ is the subvector of $\mathbf{x}_{n_{2}}^{(2)}$ obtained by taking the entries with row indices belonging to $\overrightarrow{\mathcal{A}}^{(2)}$ from $\mathbf{x}_{n_{2}}^{(2)}$. The combined demodulated signal from both subbands for interference cancellation can be written as:

$$
\tilde{\mathbf{y}}=\boldsymbol{\Xi} \tilde{\mathbf{x}}+\tilde{\mathbf{w}}
$$

where $\tilde{\mathbf{w}}=\left[\left(\tilde{\mathbf{w}}^{(1)}\right)^{T}\left(\tilde{\mathbf{w}}^{(2)}\right)^{T}\right]^{T}$ denotes the effective noise including both the residual INI and AWGN, and

$$
\boldsymbol{\Xi}=\left[\begin{array}{cc}
\rho^{(1)} \mathbf{H}_{\text {sub }}^{(1)} & \mathbf{E}_{(1,2)}^{I C} \\
\mathbf{E}_{(2,1)}^{I C} & \rho^{(2)} \mathbf{H}_{\text {sub }}^{(2)}
\end{array}\right] .
$$

In (39), $\mathbf{H}_{\text {sub }}^{(1)} \in \mathbb{C}^{N_{\mathcal{A}}^{(1)} \times N_{\mathcal{A}}^{(1)}}$ is the diagonal CFR matrix constructed by taking the diagonal elements of $\mathbf{H}_{1}^{(1)}$ with indices from $\mathcal{A}^{(1)} . \mathbf{H}_{\text {sub }}^{(2)}$ is the $n_{2} N_{\mathcal{A}}^{(2)}$-dimensional diagonal CFR matrix formed by taking the diagonal entries of $\mathbf{H}_{n_{2}}^{(2)}$ with indices belonging to $\overrightarrow{\mathcal{A}}^{(2)}$. $\mathbf{E}_{(1,2)}^{I C} \in \mathbb{C}^{N_{\mathcal{A}}^{(1)} \times\left(n_{2} N_{\mathcal{A}}^{(2)}\right)}$ is the submatrix of $\mathbf{E}_{(1,2)}$, which is formed by taking the entries of $\mathbf{E}_{(1,2)}$ with row indices belonging to $\mathcal{A}^{(1)}$ and column indices belonging to $\overrightarrow{\mathcal{A}}^{(2)} . \mathbf{E}_{(2,1)}^{I C} \in \mathbb{C}^{\left(n_{2} N_{\mathcal{A}}^{(2)}\right) \times N_{\mathcal{A}}^{(1)}}$ is formed by taking the entries of $\mathbf{E}_{(2,1)}$ with row indices belonging to $\overrightarrow{\mathcal{A}}^{(2)}$ and column indices belonging to $\mathcal{A}^{(1)}$. For $\tilde{\mathbf{w}} \in \mathbb{C}^{\tilde{L} \times 1}, \tilde{\mathbf{w}}^{(1)} \in \mathbb{C}^{N_{\mathcal{A}}^{(1)} \times 1}$ and $\tilde{\mathbf{w}}^{(2)} \in \mathbb{C}^{n_{2} N_{\mathcal{A}}^{(2)} \times 1}$ denote the residual INI and AWGN for subband 1 and subband 2 , respectively. The variance vector of $\tilde{\mathbf{w}}^{(1)}$ is $\tilde{\boldsymbol{\gamma}}^{(1)}=\left[\tilde{\sigma}_{(1)}^{2}(0), \cdots, \tilde{\sigma}_{(1)}^{2}\left(N_{\mathcal{A}}^{(1)}-1\right)\right]^{T}$. The $k$ th element of $\tilde{\gamma}^{(1)}$ can be written as

$$
\tilde{\sigma}_{(1)}^{2}(k)=\sigma_{(1)}^{2}\left[\mathcal{A}^{(1)}(k)\right]+\varepsilon_{R e}^{(1)}\left[\mathcal{A}^{(1)}(k)\right], 0 \leq k<N_{\mathcal{A}}^{(1)},
$$

with $\varepsilon_{R e}^{(1)}\left[\mathcal{A}^{(1)}(k)\right]=\sum_{u=0, u \notin \mathcal{A}(2)}^{Z^{(2)}-1} P_{u}^{(1)}\left[\mathcal{A}^{(1)}(k)\right]$ being the residual INI power. Similarly, the variance vector of $\tilde{\mathbf{w}}^{(2)}$ is $\tilde{\gamma}_{n_{2}}^{(2)}=$ $\mathbf{1}_{n_{2}} \otimes \tilde{\boldsymbol{\gamma}}^{(2)}$ with $\tilde{\boldsymbol{\gamma}}^{(2)}=\left[\tilde{\sigma}_{(2)}^{2}(0), \cdots, \tilde{\sigma}_{(2)}^{2}\left(N_{\mathcal{A}}^{(2)}-1\right)\right]^{T}$. The $k$ th element of $\tilde{\gamma}^{(2)}$ can be obtained as

$$
\tilde{\sigma}_{(2)}^{2}(k)=\sigma_{(2)}^{2}\left[\mathcal{A}^{(2)}(k)\right]+\varepsilon_{R e}^{(2)}\left[\mathcal{A}^{(2)}(k)\right], 0 \leq k<N_{\mathcal{A}}^{(2)},
$$

where

$$
\varepsilon_{R e}^{(2)}\left[\mathcal{A}^{(2)}(k)\right]=\sum_{u^{\prime}=0, u^{\prime} \notin \mathcal{A}^{(1)}}^{Z^{(1)}-1} P_{u^{\prime}}^{(2)}\left[\mathcal{A}^{(2)}(k)\right]
$$

is the effective noise variance induced by the non-dominant interfering subcarriers. The covariance matrix of zero-mean effective noise vector $\tilde{\mathbf{w}}$ can be written as

$$
\boldsymbol{\Gamma}=\operatorname{diag}\left(\left[\left(\tilde{\boldsymbol{\gamma}}^{(1)}\right)^{\mathrm{T}}\left(\tilde{\boldsymbol{\gamma}}_{\mathbf{n}_{2}}^{(2)}\right)^{\mathrm{T}}\right]^{\mathrm{T}}\right) .
$$

Based on (38), a soft-output OSIC algorithm is proposed for joint detection of the major victim subcarriers and interfering subcarriers. Since the detection order has a major impact on the OSIC detection, the symbol with highest post-detection signal-to-interference and noise power ratio (SINR) is detected first to reduce the error propagation [28]. The proposed softoutput minimum mean square error (MMSE) OSIC receiver consists of $\tilde{L}$ stages. Let us assume the symbols with indexes $\left[\mathcal{J}_{1}, \mathcal{J}_{2}, \cdots, \mathcal{J}_{l-1}\right]$ have been detected. For the $l$ th stage, the MMSE filter matrix $\mathbf{W}_{l}$ is given by

$$
\mathbf{W}_{l}=\left(\boldsymbol{\Xi}_{l}\right)^{H}\left[\boldsymbol{\Xi}_{l}\left(\boldsymbol{\Xi}_{l}\right)^{H}+\boldsymbol{\Gamma}\right]^{-1},
$$


where $\boldsymbol{\Xi}_{l} \in \mathbb{C}^{\tilde{L} \times(\tilde{L}-l+1)}$ is the matrix generated by removing the columns $\left\{\boldsymbol{\Xi}\left(:, \mathcal{J}_{1}\right), \cdots, \boldsymbol{\Xi}\left(:, \mathcal{J}_{l-1}\right)\right\}$ from $\boldsymbol{\Xi}$. For the first stage, $\boldsymbol{\Xi}_{1}=\boldsymbol{\Xi}$. The SINR for the undetected layers is

$$
\operatorname{SINR}(t)=\frac{\left|\mu_{t}\right|^{2}}{\xi_{t}}, t=1, \cdots, \tilde{L}-l+1,
$$

where

$$
\begin{aligned}
\mu_{t} & =\mathbf{W}_{l}(t,:) \boldsymbol{\Xi}_{l}(: t), \\
\xi_{t} & =\sum_{t^{\prime} \neq t}\left|\mathbf{W}_{l}(t,:) \boldsymbol{\Xi}_{l}\left(:, t^{\prime}\right)\right|^{2}+\mathbf{W}_{l}(t,:) \boldsymbol{\Gamma} \mathbf{W}_{l}(t,:)^{H} .
\end{aligned}
$$

The layer with maximum $\operatorname{SINR}(t), t=1, \cdots, \tilde{L}-l+1$, is chosen and detected as follows

$$
\begin{aligned}
t_{l} & =\arg \max _{t} \operatorname{SINR}(t), \\
\hat{x}_{t_{l}} & =\mathbf{W}_{l}\left(t_{l},:\right) \tilde{\mathbf{y}}_{l},
\end{aligned}
$$

where $\tilde{\mathbf{y}}_{l}$ is the updated received vector after cancellation of $l-1$ previous detected symbols [28]. With (46) and (47), we can regard $\hat{x}_{t_{l}}$ as an observation of $x_{t_{l}}$ as

$$
\hat{x}_{t_{l}}=\mu_{t_{l}} x_{t_{l}}+n_{l},
$$

where $n_{l}$ is a zero-mean Gaussian noise with variance $\xi_{t_{l}}$. Assume that $\hat{x}_{t_{l}}$ corresponds to the $\mathcal{J}_{l}$ th element of $\tilde{\mathbf{x}}$ and it is mapped from the coded bit vector $\mathbf{b}_{\mathcal{J}_{l}}$. Using Bayes' rule and max-log approximation [29], the LLR value for the $j$ th bit of $\mathbf{b}_{\mathcal{J}_{l}}$ can be calculated as

$$
\begin{aligned}
& L\left[\mathbf{b}_{\mathcal{J}_{l}}(j)\right]= \ln \frac{\sum_{x_{t_{l}} \in \beta_{j}^{+}} f\left(x_{t_{l}} \mid \hat{x}_{t_{l}}\right)}{\sum_{x_{t_{l}} \in \beta_{j}^{-}} f\left(x_{t_{l}} \mid \hat{x}_{t_{l}}\right)} \\
& \approx \frac{1}{\xi_{t_{l}}}\left[\min _{x_{t_{l}} \in \beta_{j}^{-}}\left|\hat{x}_{t_{l}}-\mu_{t_{l}} x_{t_{l}}\right|^{2}\right. \\
& \\
&\left.\quad-\min _{x_{t_{l}} \in \beta_{j}^{+}}\left|\hat{x}_{t_{l}}-\mu_{t_{l}} x_{t_{l}}\right|^{2}\right],
\end{aligned}
$$

where $f\left(x_{t_{l}} \mid \hat{x}_{t_{l}}\right)$ denotes the posterior probability density function (PDF) of $x_{t_{l}}, \beta_{j}^{+}$and $\beta_{j}^{-}$denote the subset of constellation symbols with the $j$ th bit being +1 and -1 , respectively. Using (49), $\tilde{\mathbf{y}}_{l}$ is updated through interference cancellation for the next stage as

$$
\tilde{\mathbf{y}}_{l+1}=\tilde{\mathbf{y}}_{l}-\mathbf{\Xi}_{l}\left(:, t_{l}\right) \operatorname{slice}\left(\hat{x}_{t_{l}}\right),
$$

where $\operatorname{slice}(x)$ denote the constellation symbol which is nearest to $x$.

For those subcarriers belonging to $\mathcal{A}_{c}^{(i)}$, i.e., the complement of $\mathcal{A}^{(i)}, i=1,2$, all the INI are regarded as the effective noise. Based on (20), the demodulated signal can be rewritten as

$$
\hat{y}_{k, m}^{(i)}=\rho^{(i)} x_{k, m}^{(i)} H^{(i)}(k)+I(k), k \in \mathcal{A}_{c}^{(i)},
$$

where $I(k)$ denotes the zero-mean compound noise whose variance is summation of AWGN variance and the interference variance, i.e., $\hat{\sigma}_{(i)}^{2}(k)=\sigma_{(i)}^{2}(k)+\varepsilon_{\text {eff }}^{(i)}(k), i=1,2$, and $\varepsilon_{e f f}^{(i)}(k)=P_{I}^{(i)}(k)=\sum_{u=0}^{Z^{(j)}-1} P_{u}^{(i)}(k), i \neq j$. Suppose $b_{k, m}^{(i)}(l)$ is the $l$ th bit of $\mathbf{b}_{k, m}^{(i)}$ which is mapped to constellation symbol $x_{k, m}^{(i)}$. Using Bayes' rule and max-log approximation [29], the
LLR of $b_{k, m}^{(i)}(l)$ can be calculated as

$$
\begin{aligned}
& L\left(b_{k, m}^{(i)}(l)\right)= \ln \frac{\sum_{x_{k, m}^{(i)} \in \beta_{l}^{+}} f\left(x_{k, m}^{(i)} \mid \hat{y}_{k, m}^{(i)}\right)}{\sum_{x_{k, m}^{(i)} \in \beta_{l}^{-}} f\left(x_{k, m}^{(i)} \mid \hat{y}_{k, m}^{(i)}\right)} \\
& \approx \frac{1}{\hat{\sigma}_{(i)}^{2}(k)}\left[\min _{x_{k, m}^{(i)} \in \beta_{l}^{-}}\left|\hat{y}_{k, m}^{(i)}-\rho^{(i)} x_{k, m}^{(i)} H^{(i)}(k)\right|^{2}\right. \\
&\left.\quad-\min _{x_{k, m}^{(i)} \in \beta_{l}^{+}}\left|\hat{y}_{k, m}^{(i)}-\rho^{(i)} x_{k, m}^{(i)} H^{(i)}(k)\right|^{2}\right],
\end{aligned}
$$

where $f\left(x_{k, m}^{(i)} \mid \hat{y}_{k, m}^{(i)}\right)$ denotes the posterior PDF of $x_{k, m}^{(i)}$, $\beta_{l}^{+}$and $\beta_{l}^{-}$denote the subset of constellation symbols with $b_{k, m}^{(i)}(l)=+1$ and $b_{k, m}^{(i)}(l)=-1$, respectively.

The proposed INI cancellation scheme is summarized as follows:

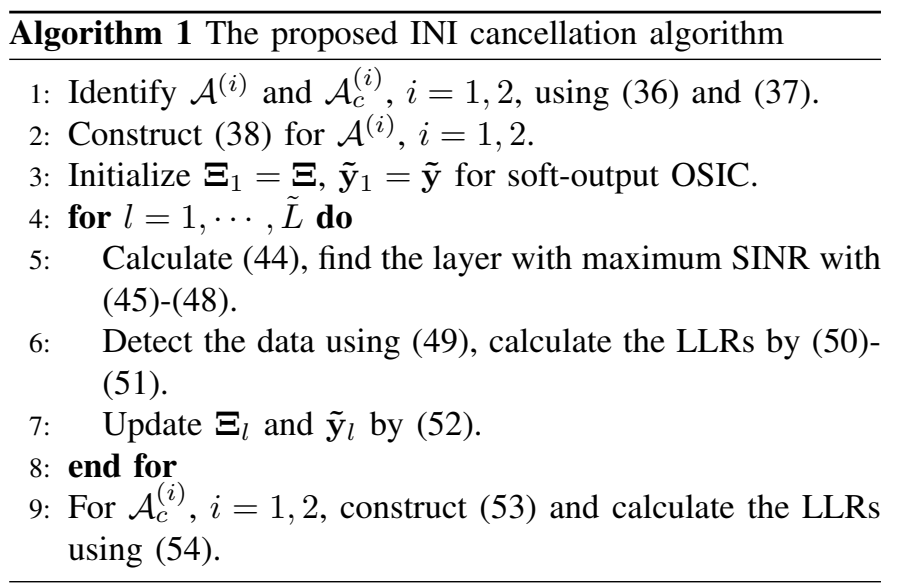

We briefly evaluate the computational complexity of the proposed INI cancellation scheme. Considering the number of complex multiplications (CM) as a complexity metric, the product of an $m \times r$ matrix with an $r \times n$ matrix requires $m r n$ operations, and the inversion of an $n \times n$ matrix requires $\mathcal{O}\left(n^{3}\right)$ operations. Since the complexity of matrix inversion depends on the specific implemented algorithm, here we take the typical value $\frac{8}{3} n^{3}$ CMs [30] for the complexity analysis. For the subcarriers belonging to $\mathcal{A}^{(i)}, i=1,2, \tilde{L}$ iterations are executed for the soft-output OSIC algorithm. During the $l$ th OSIC iteration, the calculation of (44) requires two matrix multiplications and one matrix inversion which requires $\left(\frac{14}{3}\right) \tilde{L}^{3}-2(l-1) \tilde{L}^{2}$ operations; the computational cost of SINR calculation in (45)(48) is $\tilde{L}(\tilde{L}-l+1)^{2}+(\tilde{L}-l+1)(2 \tilde{L}-l+1) \mathrm{CMs}$; the symbol detection and interference cancellation for (49) and (52) need $2 \tilde{L}$ CMs. For the LLRs calculation in both (51) and (54), a simplified LLR demapper scheme can be adopted which only requires $2 \mathrm{CMs}$ for each complex symbol [29]. Therefore, the total complexity for the soft-output OSIC is approximately $\mathcal{O}\left(5 \tilde{L}^{4}\right)$. Except the subcarriers used for softoutput OSIC, the detection of other $\tilde{L}_{c}=Z^{(1)}+n_{2} Z^{(2)}-\tilde{L}$ subcarriers belonging to $\mathcal{A}_{c}^{(i)}, i=1,2$, requires the complexity of $\mathcal{O}\left(2 \tilde{L}_{c}\right)$.

Note that the proposed INI cancellation scheme has adapt- 
ability to different system settings and INI levels. When GB is sufficient, the INI may be trivial which leads to the empty $\mathcal{A}^{(i)}, i=1,2$, all the INI can be regarded as effective noise. On the other hand, for those systems using higher modulation order or small GB, SIR threshold can be reduced to use more subcarriers to do the joint interference cancellation to maintain the system performance with the cost of higher complexity.

\section{SIMULATION RESULTS AND DISCUSSIONS}

In this section, we evaluate the accuracy of the derived analytical expression for INI power and the performance of the proposed INI cancellation algorithm for W-OFDM systems. Two adjacent subbands with different type of numerologies illustrated in Fig. 2 are considered. The first subband adopts subcarrier spacing $\Delta f^{(1)}=15 \mathrm{kHz}$ and 1024-length DFT, while the second subband has $\Delta f^{(2)}=30 \mathrm{kHz}$ with 512 length DFT or $\Delta f^{(2)}=60 \mathrm{kHz}$ with 256-length DFT. We define $\mu_{C P}=N_{C P}^{(i)} / N^{(i)}, \mu_{T x}^{(i)}=N_{T x_{-} \text {roff }}^{(i)} / N^{(i)}$, and $\mu_{R x}^{(i)}=N_{R x_{\_} \text {roff }}^{(i)} / N^{(i)}, i=1,2$, to describe the length of $\mathrm{CP}$, roll-off lengths of transmit window and receive window, respectively. $\mu_{C P}$ is set to $7 \%$, e.g., 72 samples for length1024 OFDM. The RC windows are used for both transmitter and receiver. One RB (resource block) has 12 subcarriers.

\section{A. INI Analysis}

Fig. 7 compares the simulated SIR and analytical SIR versus subcarrier indexes with different subcarrier spacing. The GB between the two subbands is set as $0, \Delta f^{(2)}$ and $2 \Delta f^{(2)}$, respectively. In this study, we assume $\Delta f^{(2)}=30 \mathrm{kHz}$, both subbands contain one RB, the roll-off lengths for transmitter window and receiver window are set to be $4 \%$ and $1 \%$, respectively. The signal is modulated using quadrature phase shift keying (QPSK) with power normalized to unity. The Pedestrian-A channel model [31] is considered.

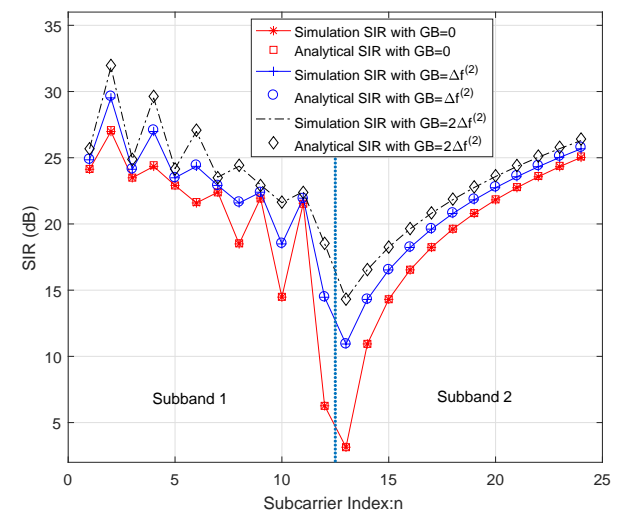

Fig. 7. SIR for the adjacent subbands.

It can be seen in Fig. 7 that there is a good match between the theoretical SIRs and numerical SIRs, which validate our analysis in Section IV. It is also observed that as the GB increases, the SIRs of both subbands increase accordingly. In all cases with different GB values, the edge subcarrier of subbands 2 (the subcarrier with index 13) with larger subcarrier spacing has the smallest SIRs, which may lead to worse bit error rate (BER) performance. In general, those subcarriers with larger spectral distance from the interfering subband have higher SIRs, since the INI power depends on the DFT magnitude of overlapping windows at the spectral distance. Meanwhile, the SIR curves of subband 1 with small subcarrier spacing have some fluctuations as spectral distance increases, as shown in Fig. 7. These properties provide useful guidance in system design. For example, we can choose the subcarrier positions with high SIRs for reference signals; modulation and coding parameters can be set adaptively based on the SIRs.

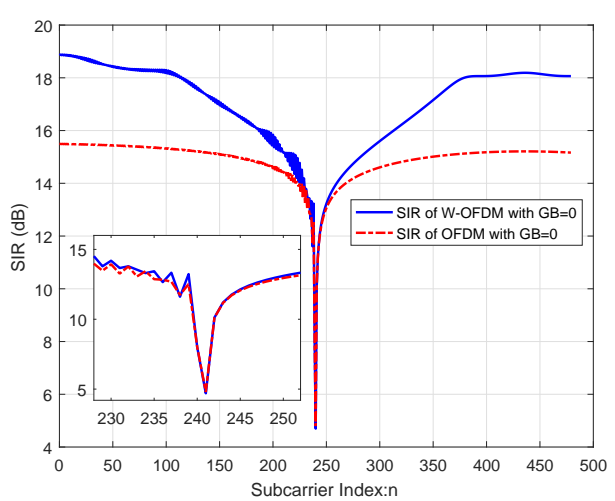

Fig. 8. SIR comparison for W-OFDM and OFDM systems.

In Fig. 8, we compare the SIRs for OFDM systems and WOFDM systems when no GB is used. In this simulation, both subbands have 20 RBs. It can be observed from Fig. 8 that the SIR of W-OFDM is significantly larger than that of OFDM for most of the RBs far away from the edge. Specifically, for the subband 1 with small subcarrier spacing, when the subcarrier index is smaller than 148 , i.e., the distance to the edge subcarrier of subband 2 is larger than $93 \Delta f^{(1)}$, the SIR gain of W-OFDM is larger than $2 \mathrm{~dB}$ compared to OFDM. Similarly, for subband 2 with large subcarrier spacing, when the subcarrier index is larger than 345, the SIR gain of WOFDM is larger than $2 \mathrm{~dB}$. The SIR gain of W-OFDM comes from the smooth transition edges of overlapping windows, which leads to more rapid decrease of INI compared to the rectangular overlapping windows for OFDM. However, we can also find that the edge RBs of W-OFDM still suffer severe INI similar to OFDM, since the average SIR for the edge RBs (the subcarriers with index 229-240 for subband 1 and subcarriers with index 241-252 for subband 2) for W-OFDM is only 0.19 $\mathrm{dB}$ higher than that of the edge RBs of OFDM systems. This result, on one hand, confirms that when there is non-sufficient $\mathrm{GB}$, the spectral distance is the major impact factor on the INI level compared with the roll-off length of the window, on the other hand, necessitates the INI cancellation algorithm for the edge RBs.

It has been mentioned in the literatures [23], [27], the increase of roll-off length of transmit window leads to better spectral containment and the larger roll-off length of receive window offers benefit for rejecting adjacent channel interference. However, to avoid ISI and keep a fixed CP overhead, 


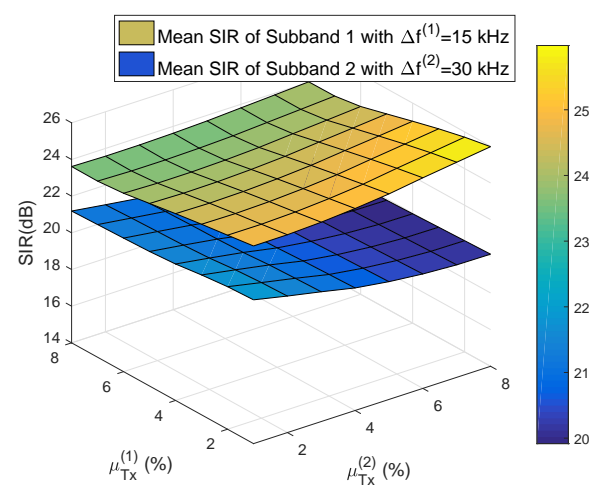

(a) $\Delta f^{(1)}=15 \mathrm{kHz}, \Delta f^{(2)}=30 \mathrm{kHz}$.

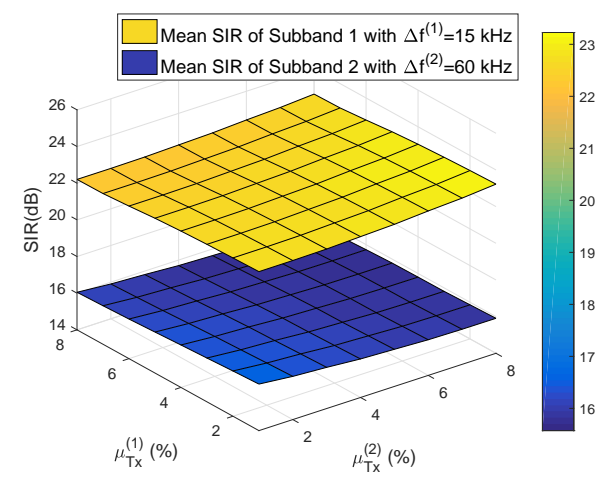

(b) $\Delta f^{(1)}=15 \mathrm{kHz}, \Delta f^{(2)}=60 \mathrm{kHz}$.

Fig. 9. SIRs for different transmit window roll-off length and receiver window roll-off length.

increasing the roll-off length of transmit window leads to decreasing the roll-off length of receive window. In order to measure how the roll-off lengths of the windows affect the INI level, we evaluate the average SIR of two subbands under different values of $\mu_{T x}^{(1)}$ and $\mu_{T x}^{(2)}$ in Fig. 9. Here, we set $\mu_{T x}^{(i)}+\mu_{R x}^{(i)}=7 \%$ for $i=1,2$, and GB $=60 \mathrm{kHz}$ for moderate INI scenario. The subcarrier spacings $\Delta f^{(1)}=15$ kHz, $\Delta f^{(2)}=30 \mathrm{kHz}$ are used in Fig. $9\left(\right.$ a) and $\Delta f^{(1)}=15$ kHz, $\Delta f^{(2)}=60 \mathrm{kHz}$ are adopted in Fig. 9(b).

As shown in Fig. 9, subband 1 with small subcarrier spacing has larger average SIR than that of subband 2 with large subcarrier spacing for all the different parameters settings. In the simulation, the average SIR of subband 1 with $\Delta f^{(1)}=15$ $\mathrm{kHz}$ is $3.79 \mathrm{~dB}$ larger than that of subband with $\Delta f^{(2)}=30$ $\mathrm{kHz}$, and is $6.68 \mathrm{~dB}$ larger than that of the subband with $\Delta f^{(2)}=60 \mathrm{kHz}$. Compare Fig. 9(a) and Fig. 9(b), we can find that when the subcarrier spacing difference increases, the average SIRs for both subbands deteriorates accordingly. The average SIRs of subband 1 and subband 2 decrease by $1.73 \mathrm{~dB}$ and $4.6 \mathrm{~dB}$, respectively, when $\Delta f^{(2)} / \Delta f^{(1)}=2$ in Fig. 9(a) is changed to $\Delta f^{(2)} / \Delta f^{(1)}=4$ in Fig. 9(b). In the scenario of modest INI, both the larger roll-off length of transmit window for aggressor and larger roll-off length of receive window for victim offer some SIR gain for subband 1. In Fig. 9(a), when OFDM transmitter is adopted in subband 2 as the aggressor with $\mu_{T x}^{(2)}=0$, increasing $\mu_{R x}^{(1)}$ from $0 \%$ to $7 \%$ (i.e., decreasing $\mu_{T x}^{(1)}$ from $7 \%$ to $0 \%$ ) offers $1.2 \mathrm{~dB}$ SIR gain for subband 1 . When OFDM receiver is used for subband 1 as the victim with $\mu_{R x}^{(1)}=0 \%$ (i.e., $\mu_{T x}^{(1)}=7 \%$ ), increasing $\mu_{T x}^{(2)}$ from $0 \%$ to $7 \%$ offers $0.4 \mathrm{~dB}$ average SIR gain for subband 1 . Similar phenomenon can be observed in Fig. 9(b). For the subband 2 with large subcarrier spacing, SIR gain is noticeable with the increase of $\mu_{R x}^{(2)}$, as shown in Fig. 9(a) and Fig. 9(b). When $\mu_{T x}^{(1)}=0$, increasing $\mu_{R x}^{(2)}$ from $0 \%$ to $7 \%$ generates $1.7 \mathrm{~dB}$ SIR gain in Fig. 9(a) and $0.64 \mathrm{~dB}$ gain in Fig. 9(b) for subband 2. However, no apparent SIR gain can be found for subband 2 with the increase of $\mu_{T x}^{(1)}$. We reckon that this behavior stems from the combined effect of the following two factors. Firstly, although the increase of $\mu_{T x}^{(1)}$ may decrease the sidelobes of subband 1 as the interferer, it also generates larger overlapping of adjacent symbols which lead to extra
INI from previous LCM symbol for subband 2, as shown in Fig. 5. Secondly, for subband 2, the smooth transition edges of overlapping windows are mostly inherited from the roll-off parts of the victim's receive window.

\section{B. INI cancellation algorithm}

In this subsection, the performance of the proposed INI cancellation algorithm is evaluated. We set $\mathrm{GB}=0, \Delta f^{(1)}=$ $15 \mathrm{kHz}$ and $\Delta f^{(2)}=30 \mathrm{kHz}$. For both subbands, we set $\mu_{C P}=7 \%, \mu_{T x}^{(1)}=\mu_{T x}^{(2)}=4 \%$ and $\mu_{R x}^{(1)}=\mu_{R x}^{(2)}=1 \%$. For channel coding, a half-rate convolutional code with encoding polynomials $(7,5)_{8}$ is considered. The modulation schemes is the 16-ary quadrature amplitude modulation (16QAM) or 64QAM. We use the Pedestrian-A channel model [31] for both subbands. In the following simulation results, SoftZF refers to the zero-forcing detector generating soft bits without considering the INI [29], SoftMMSE refers to the soft-output MMSE detector which considers all the INI power as the effective noise [5]. The proposed SoftOSIC algorithm takes $\mathcal{T}_{1}=20 \mathrm{~dB}$ and $\mathcal{T}_{2}=25 \mathrm{~dB}$ to choose the severe victim subcarriers and dominant interfering subcarriers. The soft information generated by detectors is fed forward to the channel decoder for soft decoding.

In Fig. 10, we compare the mean square error (MSE) per-

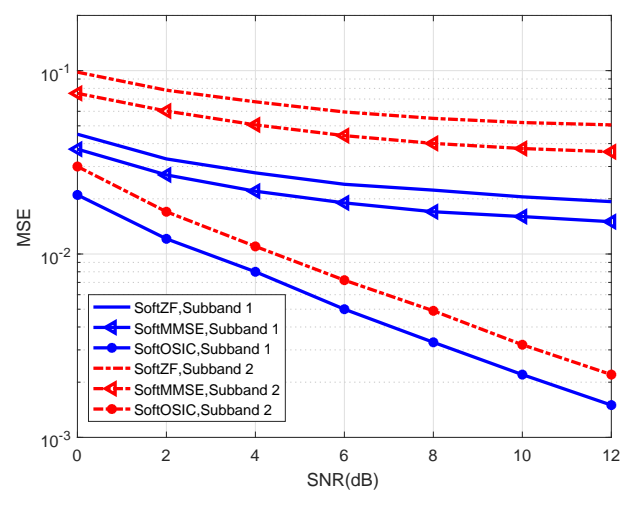

Fig. 10. MSE versus SNR for different detectors with 16QAM.

formance of different detection algorithms for both subbands 


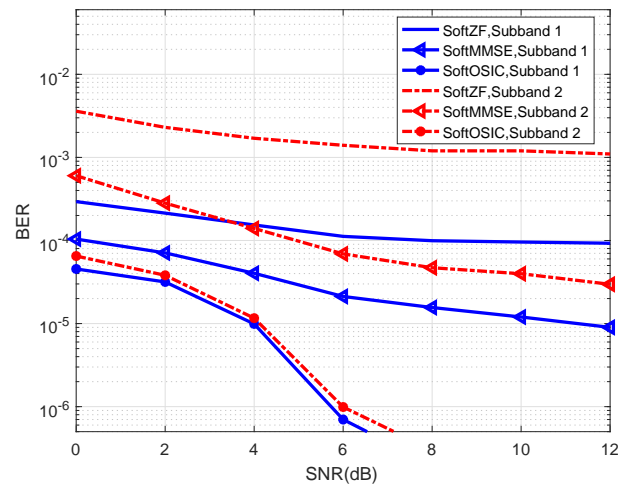

Fig. 11. BER versus SNR for different detectors with 16QAM.

with 16QAM modulation. It can be seen from Fig. 10, the MSE performance of subband 1 with small subcarrier spacing is always better than that of subband 2. This follows from the fact that the edge subcarrier of subband 2 has the worst SIR and the average SIR of subband 2 is smaller than that of subband 1, which has been shown in Fig. 7 and Fig. 9(a), respectively. The proposed SoftOSIC detector has the better MSE performance than the SoftZF and SoftMMSE detectors, because the joint interference cancellation is executed for both the victim subcarriers with severe INI and the dominant interfering subcarriers. The SoftMMSE detector can offer some performance gain compared with SoftZF detector, since the INI power is taken into account as the effective noise.

In Fig. 11, the BER vs. SNR performance is presented for receivers using different detectors. We observe that the performance of the receiver is significantly improved by the SoftOSIC detector. For example, when SNR $=6 \mathrm{~dB}$, the proposed SoftOSIC can achieve BER $\leq 10^{-6}$ for both subbands, while the BER of SoftMMSE is larger than $10^{-5}$ and BER of SoftZF is larger than $10^{-4}$ for both subbands. Since the dominant INI for both subbands is cancelled during the OSIC process, the difference of BER performances for both subbands is very small with the proposed receiver.

To evaluate the system performance for high order modulation, MSE and BER performance for receivers using different detectors are shown in Fig. 12 and Fig. 13, respectively, when the signal is modulated by 64QAM with power normalized to unity for both subbands.

It can be observed form Fig. 12 that the increase of SNR does not bring noticeable MSE performance improvements for both SoftZF and SoftMMSE schemes even at high SNRs. This is because the effect of INI becomes dominant factor to the system performance compared with the AWGN for the scenarios considering higher order constellations. This leads to an error floor of BER performance for both SoftZF and SoftMMSE, as shown in Fig. 13. On the contrary, both the MSE and BER performances of the proposed SoftOSIC scheme improve significantly as the SNR increases. The proposed SoftOSIC algorithm can achieve better MSE performance compared with that of SoftZF and SoftMMSE. When SNR is $14 \mathrm{~dB}$, the BERs of both subbands are below $10^{-6}$ for the SoftOSIC algorithm, while BERs of SoftMMSE algorithm are larger than $8.9 \times 10^{-5}$, and BERs of the SoftZF algorithm are larger than $10^{-3}$ for both subbands.

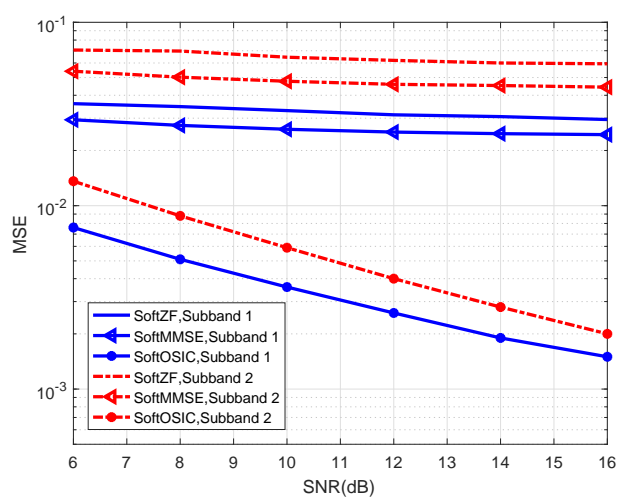

Fig. 12. MSE versus SNR for different detectors with 64QAM.

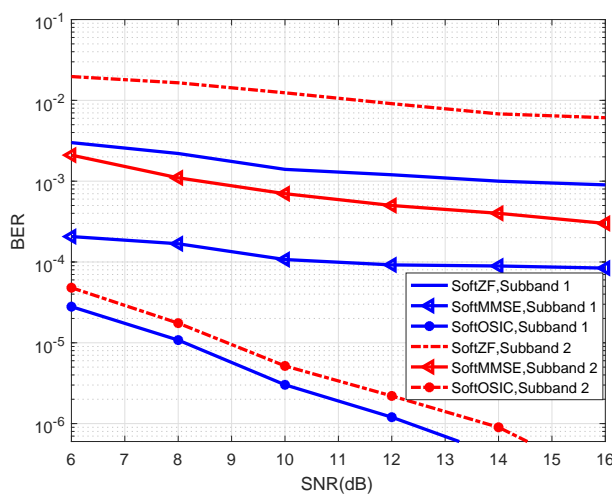

Fig. 13. BER versus SNR for different detectors with 64QAM.

\section{CONCLUSIONS}

In this paper, we investigate the INI modeling in the scenario of mixed numerologies transmission for W-OFDM systems. Analytical expressions of the INI power are derived and verified for the subbands using different numerologies under the frequency selective fading channels. The derived INI model and INI power analytical expressions can be straightforwardly applied to OFDM system and can be extended to the other subcarrier-specific windowed waveforms to assist in system design and waveform optimization for the next generation wireless communications. Based on the proposed analytical expressions and the derived INI properties, a novel INI cancellation scheme is proposed for the receiver, which divides the INI into dominant part and the effective noise part. A soft-output OSIC algorithm is performed to cancel the dominant INI while the residual INI are regarded as effective noise to affect the LLRs calculation for coded bits. Various simulation results have been presented to show that the proposed algorithm has better performance than the existing algorithms considered. It is worth noting that besides the generalized synchronous scenario considered in this paper, asynchronous mixed numerologies transmission is also of practical importance. This will be the focus of our future study. 


\section{APPENDIX A \\ DERIVATION OF (9)}

Substitute (3) and (1) into (4) and assume that the transmitter window and the receiver window satisfy conditions (6) and (7), we have

$$
\begin{gathered}
\hat{y}_{k, m}^{(i)}=\sum_{n} \sum_{l=0}^{L_{C H}^{(i)}-1} h^{(i)}(l) \sum_{u=0}^{Z^{(i)}-1} \sum_{v=-\infty}^{\infty} x_{u, v}^{(i)} g_{u, v}^{(i)}(n-l) \overline{q_{k, m}^{(i)}(n)}+w^{(i)}(k) \\
=\frac{1}{N^{(i)}} \sum_{u=0}^{Z^{(i)}-1} x_{u, v}^{(i)} \sum_{l=0}^{L_{C H}^{(i)}-1} h^{(i)}(l) e^{-j \frac{2 \pi}{N^{(i)}}\left(u+\Delta F^{(i)}\right) l} \sum_{n} g_{u}^{(i)}(n-l) \\
\times q_{k}^{(i)}(n) e^{-j \frac{2 \pi}{N^{(i)}}(k-u)\left(n-N_{C P}^{(i)}\right)}+w^{(i)}(k) \\
=\frac{1}{N^{(i)}} \sum_{u=0}^{Z^{(i)}-1} x_{u, m}^{(i)} H^{(i)}(u) \sum_{n^{\prime}=0}^{N_{T}^{(i)}-1} q_{k}^{(i)}\left(n^{\prime}\right) e^{j \frac{2 \pi}{N^{(i)}}(u-k)\left(n^{\prime}-N_{C P}^{(i)}\right)} \\
+w^{(i)}(k) .
\end{gathered}
$$

Substituting (8) to (55), we obtain (9).

\section{APPENDIX B}

\section{DERIVATIONS OF (28)}

Substitute (2) and (5) into (26) and define $\rho_{N}^{(2)}=$ $\left(\frac{\rho^{(2)}}{\sqrt{N^{(1)} N^{(2)}}}\right)^{2}$, we have

$$
\begin{aligned}
& P_{u}^{(1)}(k, m) \\
& =\rho_{N}^{(2)} \sum_{v=-\infty}^{\infty} \mid \sum_{l=0}^{L_{C H}^{(2)}-1} h^{(2)}(l) e^{-j \frac{2 \pi}{N(2)}\left(u+\Delta F^{(2)}\right)(l)} \\
& \quad \times \sum_{n} g_{u}^{(2)}\left(n-v N_{T}^{(2)}-l\right) q_{k}^{(1)}\left(n-m N_{T}^{(1)}\right) \\
& \quad \times\left. e^{j \frac{2 \pi\left(u+\Delta F^{(2)}\right)}{N^{(2)}}\left(n-N_{C P}^{(2)}-v N_{T}^{(2)}\right)} e^{-j \frac{2 \pi k}{N^{(1)}}\left(n-N_{C P}^{(1)}-m N_{T}^{(1)}\right)}\right|^{2} \\
& \stackrel{(a)}{\approx} \rho_{N}^{(2)} \sum_{v=-\infty}^{\infty} \mid H^{(2)}(u) \sum_{n} g_{u}^{(2)}\left(n-v N_{T}^{(2)}\right) q_{k}^{(1)}\left(n-m N_{T}^{(1)}\right) \\
& \stackrel{(b)}{=} \rho_{N}^{(2)}\left|H^{(2)}(u)\right|^{2} \sum_{d=-1}^{n_{2}-1} \mid \sum_{n} g_{u}^{(2)}\left(n-m N_{T}^{(1)}+N_{T}^{(2)}\right) \\
& \quad \times\left. q_{k}^{(1)}\left(n-m N_{T}^{(1)}\right) e^{-j \frac{2 \pi}{N(1)}[k-\hat{k}(u)](n)}\right|^{2} \\
& =\rho_{N}^{(2)}\left|H^{(2)}(u)\right|^{2} \sum_{d=-1}^{n_{2}-1}\left|\sum_{n} g_{u}^{(2)}\left(n-d N_{T}^{(2)}\right) q_{k}^{(1)}(n) e^{-j \frac{2 \pi}{N(1)} \Delta k^{(1)} n}\right|^{2} \\
& =\rho_{N}^{(2)}\left|H^{(2)}(u)\right|^{2} \sum_{d=-1}^{n_{2}-1}\left|\sum_{n} \Omega_{g_{u}^{(2)}, d N_{T}^{(2)}}^{q^{(1)}, 0}(n) e^{-j \frac{2 \pi}{N^{(1)}} \Delta k^{(1)} n}\right|^{2}
\end{aligned}
$$

In (56), approximate equality (a) follows the assumption that the channel is weakly or mildly time-dispersive, thus $g_{u}^{(2)}\left(n-v N_{T}^{(2)}-l\right) \approx g_{u}^{(2)}\left(n-v N_{T}^{(2)}\right)$; equality (b) holds due to $n_{2} N_{T}^{(2)}=N_{T}^{(1)}$ and only those transmit windows $g_{k}^{(2)}\left(n-v N_{T}^{(2)}\right)$ with $v=m n_{2}-1, m n_{2}, m n_{2}+1, \cdots, m n_{2}+$ $n_{2}-1$, may possibly overlap with the given receive window $q_{k}^{(1)}\left(n-m N_{T}^{(1)}\right)$ for numerology 1.

\section{APPENDIX C}

DERIVATIONS OF (29)

In the case of OFDM, there are $\alpha=\left\lfloor N^{(1)} / N_{T}^{(2)}\right\rfloor$ rectangular overlapping windows with length $N_{T}^{(2)}$, and one rectangular window has the length $N^{(1)}-\alpha N_{T}^{(2)}$. We use $\rho_{N}^{(2)}$ to denote $\left(\frac{\rho^{(2)}}{\sqrt{N^{(1)} N^{(2)}}}\right)^{2}$. Based on (28), we have

$$
\begin{aligned}
& \breve{P}_{u}^{(1)}(k) \\
& \approx \rho_{N}^{(2)}\left|H^{(2)}(u)\right|^{2} \sum_{d=-1}^{n_{2}-1}\left|\sum_{n} g_{u}^{(2)}\left(n-d N_{T}^{(2)}\right) q_{k}^{(1)}(n) e^{-j \frac{2 \pi}{N^{(1)}} \Delta k^{(1)} n}\right|^{2} \\
& =\rho_{N}^{(2)}\left|H^{(2)}(u)\right|^{2}\left[\left|\frac{\sin \left(\frac{\pi}{N^{(1)}} \Delta k^{(1)} \alpha N_{T}^{(2)}\right)}{\sin \left(\frac{\pi}{N^{(1)}} \Delta k^{(1)}\right)}\right|^{2}\right. \\
& \left.+\alpha\left|\frac{\sin \left(\frac{\pi}{N^{(1)}} \Delta k^{(1)} N_{T}^{(2)}\right)}{\sin \left(\frac{\pi}{N^{(1)}} \Delta k^{(1)}\right)}\right|^{2}\right]
\end{aligned}
$$

\section{APPENDIX D}

DERIVATIONS OF (31)

Substitute (2) and (5) into (30) and define $\rho_{N}^{(1)}=$ $\left(\frac{\rho^{(1)}}{\sqrt{N^{(1)} N^{(2)}}}\right)^{2}$, we have

$$
\begin{aligned}
P_{u}^{(2)}(k, m) & \\
=\rho_{N}^{(1)} & \sum_{v=-\infty}^{\infty} \mid \sum_{n} \sum_{l=0}^{L_{C H}^{(1)}-1} h^{(1)}(l) g_{u}^{(1)}\left(n-l-v N_{T}^{(1)}\right) \\
& \times e^{j \frac{2 \pi}{N^{(1)}} u\left(n-l-N_{C P}^{(1)}-v N_{T}^{(1)}\right)} \\
& \times\left. q_{k}^{(2)}\left(n-m N_{T}^{(2)}\right) e^{-j \frac{2 \pi}{N^{(2)}}\left(k+\Delta F^{(2)}\right)\left(n-N_{C P}^{(2)}-m N_{T}^{(2)}\right)}\right|^{2} \\
\stackrel{(d)}{\approx} \rho_{N}^{(1)} & \sum_{v=-\infty}^{\infty} \mid \sum_{l=0}^{L_{C H}^{(1)}-1} h^{(1)}(l) e^{-j \frac{2 \pi}{N^{(1)}} u l} \sum_{n} g_{u}^{(1)}\left(n-v N_{T}^{(1)}\right) \\
& \times e^{j} \frac{2 \pi}{N^{(1)}} u\left(n-N_{C P}^{(1)}-v N_{T}^{(1)}\right) \\
& \times\left. q_{k}^{(2)}\left(n-m N_{T}^{(2)}\right) e^{-j \frac{2 \pi}{N^{(2)}}\left(k+\Delta F^{(2)}\right)\left(n-N_{C P}^{(2)}-m N_{T}^{(2)}\right)}\right|^{2} \\
\stackrel{(e)}{=} \rho_{N}^{(1)} \mid & \left.H^{(1)}(u)\right|^{2} \sum_{j=0}^{1} \mid \sum_{n} q_{k}^{(2)}\left(n-\tilde{m} n_{2} N_{T}^{(2)}-d N_{T}^{(2)}\right) \\
\times & \left.q_{k}^{(2)}\left(n-\tilde{m} n_{2} N_{T}^{(2)}-d N_{T}^{(2)}\right) e^{-j \frac{2 \pi}{N(1)}\left[n_{2}\left(k+\Delta F^{(2)}\right)-u\right] n}\right|^{2} \\
=\rho_{N}^{(1)} \mid & \left.H^{(1)}(u)\right|^{2} \sum_{j=0}^{1} \mid \sum_{n} g_{u}^{(1)}\left(n-j N_{T}^{(1)}\right) q_{k}^{(2)}\left(n-d N_{T}^{(2)}\right) \\
& \times\left. e^{-j \frac{2 \pi}{N^{(1)}} \Delta k^{(2)}(n)}\right|^{2} \\
=\rho_{N}^{(1)} \mid & \left.H^{(1)}(u)\right|^{2} \sum_{j=0}^{1}\left|\sum_{n} \Omega_{g_{u}^{(1)}}^{q_{k}^{(2)}, j N_{T}^{(2)}},-j N_{T}^{(1)}(n) e^{-j \frac{2 \pi}{N(1)} \Delta k^{(2)} n}\right|^{2}
\end{aligned}
$$

In (58), approximate equality (d), similar to approximate equality (a) in (56), follows the assumption that the channel is weakly or mildly time-dispersive, thus $g_{u}^{(1)}\left(n-l-v N_{T}^{(1)}\right) \approx$ $g_{u}^{(1)}\left(n-v N_{T}^{(1)}\right)$; equality (e) holds due to the $m$ th received symbol of subband 2 lies in the $\tilde{m}=\left\lfloor m / n_{2}\right\rfloor$ th LCM symbol, 
using $m=\tilde{m} n_{2}+d$, with $d=0,1, \cdots, n_{2}-1$, the $m$ th received symbol may possibly be interfered by the $\tilde{m}$ and $\tilde{m}-1$ th transmitted symbol of subband 1 .

\section{REFERENCES}

[1] J. G. Andrews, S. Buzzi, W. Choi, S. V. Hanly, A. Lozano, A. C. K. Soong, and J. C. Zhang," What will 5G be?" IEEE J. Select. Areas Commun., vol. 32, no.6, pp. 1065-1082, Jun. 2014.

[2] A. Ijaz, L. Zhang, M. Grau, A. Mohamed, S. Vural, A. U. Quddus, M. A. Imran, C. Foh, and R. Tafazolli,"Enabling massive IoT in 5G and beyond systems: PHY radio frame design considerations", IEEE Access, vol. 4, pp. 3322-3339, 2016.

[3] Y. Yuan and X. Zhao, "5G: Vision, scenarios and enabling technologies," ZTE Communications, vol. 13, no. 1, pp. 3-10, Mar. 2015.

[4] ITU-R, Recommendation M. 2083-0, "IMT Vision-framework and overall objectives of the future development of IMT for 2020 and beyond," ITU recommendation, Sep. 2015.

[5] L. Zhang, A. Ijaz, P. Xiao, A. Quddus, and R. Tafazolli, "Subband filtered multi-Carrier systems for multiservice wireless communications," IEEE Trans. on Wireless Commun., vol. 16, no. 3, pp. 1893-1907, Mar. 2017.

[6] A. Sahin and H. Arslan," Multi-user aware frame structure for OFDMA based system," in Proc. IEEE Vehicular Technology Conf. (VTC-Fall), Québec City, Canada, Sep. 2012, pp. 1-5.

[7] P. Guan et al., "5G field trials: OFDM-based waveforms and mixed numerologies," IEEE J. Sel. Areas Commun., , vol. 35, no. 6, pp. 12341243, Jun. 2017.

[8] Way Forward on Assumptions for Waveform Evaluation, 3GPP documant R1-163558, Huawei, HiSilicon, Busan, Korea, Apr. 2016.

[9] L. Zhang, A.Ijaz, P. Xiao, A. Quddus, and R. Tafazolli, "Single-rate and multi-rate multi-service systems for next generation and beyond communications," in Proc. IEEE Int. Symp. Pers., Indoor, Mobile Radio Commun. (PIMRC), Valencia, Spain, Sep. 2016, pp. 1-6.

[10] L. Zhang, A. Ijaz, P. Xiao, and R. Tafazolli,"Multi-service systems: An enabler of flexible 5G air-interface," IEEE Communications Magazine, to appear, 2017.

[11] E. Güvenkaya, E. Bala, R. Yang, and H. Arslan, "Time-asymmetric and subcarrier-specific pulse shaping in OFDM-based waveforms," IEEE Trans. Veh. Technol., vol.64, no. 11, pp. 5070-5082, Nov. 2015.

[12] Waveform Candidates, 3GPP documant R1-162199, Qualcomm Inc., Busan, Korea, Apr. 2016.

[13] X. Zhang, M. Jia, L. Chen, J. Ma, and J. Qiu,"Filtered-OFDM ${ }^{a}{ }^{2} E n a b l e r$ for flexible waveform in the 5th generation cellular networks," in Proc. IEEE Global Telecommun. Conf., Dec. 2015, pp. 1-6.

[14] V. Vakilian, T. Wild, F. Schaich, S.ten Brink and J.F.Frigon,"Universalfiltered multi-carrier technique for wireless systems beyond LTE," IEEE Globecom Workshops, Atlanta, USA, Dec. 2013, pp. 223-228.

[15] L. Zhang, P. Xiao, A. Zafar, A. Quddus and R. Tafazolli, "FBMC system An insight into doubly dispersive channel impact," IEEE Trans. Veh. Technol., vol. 66, no. 5, pp. 3942-3956, May, 2017.

[16] N. Michailow et al., "Generalized frequency division multiplexing for 5th generation cellular networks,"IEEE Trans. Commun., vol. 62, no. 9,pp. 3045-3061, Sep. 2014.

[17] Z. Ankarali, B. Pekoz, H. Arslan, "Flexible radio access beyond 5G: A future projection on waveform, numerology and frame design principles," IEEE Access, (accepted).

[18] RAN1 Chairman's Notes, document 3GPP TSG RAN WG1 Meeting \#86, Gothenburg, Sweden, Aug. 2016.

[19] RAN1 Chairman's Notes, document 3GPP TSG RAN WG1 Meeting \#86bis, Lisbon, Portugal, Oct. 2016.

[20] S. Y. Lien et al.," 5G new radio: Waveform, frame structure, multiple access, and initial access," IEEE Communications Magazine, vol. 55, no. 6, pp. 64-71, Jun. 2017.

[21] R. Zayani, Y. Medjahdi, H. Shaiek, and D. Roviras," WOLA-OFDM: A potential candidate for asynchronous 5G," in Proc. IEEE Globecom Workshops (GC Wkshps), Washington DC, USA, Dec. 2016, pp. 1-5.

[22] B. Farhang-Boroujeny, "OFDM versus filter bank multicarrier," IEEE Signal Process. Mag., vol. 28, no. 3, pp. 92-112, May 2011

[23] E. Bala, J. Li, and R. Yang, "Shaping spectral leakage: A novel low complexity transceiver architecture for cognitive radio," IEEE Veh. Technol. Mag., vol. 8, no. 3, pp. 38-46, Sep. 2013.

[24] E. Güvenkaya, A. Şahin, E. Bala, R. Yang, and H. Arslan, "A windowing technique for optimal time-frequency concentration and ACI rejection in OFDM-based systems," IEEE Trans. Commun., vol.63, no. 12, pp. 49774989, Dec. 2015.
[25] A. Şahin and H. Arslan, "Edge windowing for OFDM based systems," IEEE Commun. Lett., vol. 15, no. 11, pp. 1208-1211, Nov. 2011.

[26] Feasibility of Mixing Numerology in an OFDM System, 3GPP proposal, R1-163224, Ericsson, Busan, Korea, Apr. 2016.

[27] A. A. Zaidi et al., "Waveform and numerology to support 5G services and requirements," IEEE Commun. Mag.,vol. 54, no. 11, pp. 90-98, Nov. 2016.

[28] P. W. Wolniansky, G. J. Foschini, G. D. Golden, and R. A. Valenzuela, "V-BLAST: An architecture for realizing very high data rates over the rich-scattering wireless channel," in Proc. URSI Int. Symp. Signal, Syst., Electron, 1998, pp. 295-300.

[29] F. Tosato and P. Bisaglia, "Simplified soft-output demapper for binary interleaved COFDM with application to HIPERLAN/2," in Proc. IEEE Int. Conf. Commun. (ICC). Apr. 2002, pp. 664-668.

[30] V. S. Ryaben'kii and S. V. Tsynkov, A Theoretical Introduction to Numerical Analysis. Boca Raton, FL, USA: CRC Press, 2006.

[31] Evolved Universal Terrestrial Radio Access (E-UTRA); User Equipment (UE) Radio Transmission and Reception. 3GPP Specification \#: 36.101, Technical Specification Group Radio Access Network. 